\title{
MORSE INDEX COMPUTATION FOR RADIAL SOLUTIONS OF THE HÉNON PROBLEM IN THE DISK
}

\author{
ANNA LISA AMADORI, FRANCESCA DE MARCHIS, AND ISABELLA IANNI
}

\begin{abstract}
We compute the Morse index $\mathrm{m}\left(u_{p}\right)$ of any radial solution $u_{p}$ of the semilinear problem:

$$
\left\{\begin{array}{lr}
-\Delta u=|x|^{\alpha}|u|^{p-1} u & \text { in } B \\
u=0 & \text { on } \partial B
\end{array}\right.
$$

where $B$ is the unit ball of $\mathbb{R}^{2}$ centered at the origin, $\alpha \geq 0$ is fixed and $p>1$ is sufficiently large. In the case $\alpha=0$, i.e. for the Lane-Emden problem, this leads to the following Morse index formula

$$
\mathrm{m}\left(u_{p}\right)=4 m^{2}-m-2,
$$

for $p$ large enough, where $m$ is the number of nodal domains of $u$.
\end{abstract}

\section{Motivations And MAin REsults}

We consider the following classical semilinear elliptic problem

$$
\left\{\begin{array}{lr}
-\Delta u=|x|^{\alpha}|u|^{p-1} u & \text { in } B \\
u=0 & \text { on } \partial B
\end{array}\right.
$$

where $\alpha \geq 0, p>1$ and $B$ is the unit ball of $\mathbb{R}^{N}, N \geq 2$, centered at the origin.

When $\alpha>0$ (1.1) has been introduced by Hénon in 27] in the study of stellar clusters thus it is known as the Hénon problem, when $\alpha=0$ (1.1) reduces to the classical Lane-Emden problem.

From a mathematical point of view it is well known that, for any fixed $\alpha \geq 0$, problem (1.1) admits solutions, and in particular radial solutions, for every $p>1$ if $N=2$, and for every $p \in\left(1, p_{\alpha}\right)$ if $N \geq 3$, where $p_{\alpha}=\frac{N+2+2 \alpha}{N-2}$ (see 34]). Moreover for any given $m \geq 1$ there is exactly one couple of radial solutions of (1.1) which have exactly $m$ nodal zones, they are classical solutions and they are one the opposite of the other (see for instance $[12,33,30]$ ).

Observe that the two problems $(\alpha=0$ and $\alpha>0)$ have a strong correlation, indeed the change of variable

$$
v(t)=\left(\frac{2}{2+\alpha}\right)^{\frac{2}{p-1}} u(r), \quad t=r^{\frac{2+\alpha}{2}},
$$

transforms radial solutions $u$ of the Hénon problem in dimension $N$ into radial solutions $v$ of the Lane-Emden problem in dimension $M=M(N, \alpha):=\frac{2(N+\alpha)}{2+\alpha}$, with the same number of zeros. Notice that $M=N$ when $N=2$, while $M<N$ for any $N \geq 3$ and in this case $M$ may be a non integer extended dimension.

2010 Mathematics Subject classification: 35B05, 35B06, 35J91.

Keywords: superlinear elliptic boundary value problem, sign-changing radial solution, asymptotic analysis, Morse index.

The last author is partially supported by: PRIN 2017JPCAPN_003 grant, VALERE: Vain-Hopes grant, INDAM - GNAMPA. 
This paper deals with the computation of the Morse index of all the radial solutions of (1.1) in dimension $N=2$, for any $\alpha \geq 0$ fixed and for large values of the exponent $p$.

We recall that the Morse index $\mathrm{m}(u)$ of a solution $u$ of (1.1) is the maximal dimension of a subspace $X \subset H_{0}^{1}(B)$ where the quadratic form $Q_{u}: H_{0}^{1}(B) \times H_{0}^{1}(B) \rightarrow \mathbb{R}$

$$
Q_{u}(v, w)=\int_{B}\left(\nabla v \nabla w-|x|^{\alpha} p|u|^{p-1} v w\right) d x
$$

is negative definite. Equivalently, since $B$ is a bounded domain, $\mathrm{m}(u)$ can be defined as the number of the negative Dirichlet eigenvalues of the linearized operator at $u$

$$
L_{u}=-\Delta-|x|^{\alpha} p|u|^{p-1}
$$

counted with their multiplicity.

The knowledge of the Morse index has important applications: it allows to distinguish and classify solutions and to study their stability properties. Moreover it is well known that a change in the Morse index may imply bifurcation, which may also give rise to symmetry breaking phenomena ([23, 2, 5, 31, 20, ).

Focusing on radial solutions $u_{p}$ of problem (1.1), it is known, from [28, 11] in the case $\alpha=0$ and [8] in the case $\alpha>0$, that the radial Morse index $\mathrm{m}_{\mathrm{rad}}\left(u_{p}\right)$ (i.e. the number of the negative eigenvalues of $L_{u_{p}}$ in the subspace $H_{0, \text { rad }}^{1}(B)$ of the radial functions in $H_{0}^{1}(B)$ ), coincides with the number $m$ of nodal zones of $u_{p}$ :

$$
\mathrm{m}_{\mathrm{rad}}\left(u_{p}\right)=m
$$

and moreover the solution $u_{p}$ is radially nondegenerate. Nevertheless the complete Morse index of a radial solution $u_{p}$ is generally higher, and indeed the following lower bound holds true

$$
\mathrm{m}\left(u_{p}\right) \geq\left\{\begin{array}{lr}
m+(m-1) N & \text { if } \alpha \in[0,2) \\
m+(m-1)\left(N+\sum_{j=1}^{\left[\frac{\alpha}{2}\right]} N_{j+1}\right) & \text { if } \alpha \geq 2
\end{array}\right.
$$

as proved in [17] for the case $\alpha=0$ (see also [1, 10] for previous results in this direction) and then for the case $\alpha>0$ in [8] by exploiting the relation in (1.2) (see also [18]). Here $N$ stands for the dimension, $N_{j}=\frac{(N+2 j-2)(N+j-3) !}{(N-2) ! j !}$ is the multiplicity of the $j$-th eigenvalue $\lambda_{j}=j(N+j-2)$ of the Laplace-Beltrami operator on the sphere $\mathbb{S}_{N-1}$ and [.] is the integer part.

Observe that, by Morse index comparison, one deduces from the estimates (1.3) that a least energy nodal (i.e. $m \geq 2$ ) solution for problem (1.1), having Morse index 2 (cfr. [11]), can not be radial (see [8, and also [1, 10, 18).

For the Lane-Emden problem $(\alpha=0)$ and in dimension $N \geq 3$ the estimate (1.3) is surprisingly optimal, indeed in [17] the following Morse index formula has been proven:

$$
\mathrm{m}\left(u_{p}\right)=m+(m-1) N, \quad \text { for } p \in\left[\bar{p}, p_{\alpha}\right),
$$

for a certain $\bar{p}:=\bar{p}(m, N)>1$.

This formula has been then generalized to the Hénon case $(\alpha>0)$ in [6], obtaining, again in dimension $N \geq 3$, that

$$
\mathrm{m}\left(u_{p}\right)=m+(m-1) \sum_{j=1}^{\left[\frac{2+\alpha}{2}\right]} N_{j}+\sum_{j=1}^{\left\lceil\frac{\alpha}{2}\right\rceil} N_{j}
$$

for $p \in\left[\bar{p}, p_{\alpha}\right)$, where $\bar{p}:=\bar{p}(m, N, \alpha)>1$. Here [.] is the integer part and $\lceil\cdot 7$ the ceiling function. Observe that for any $\alpha>0$ the Morse index in this formula is actually higher then 
the lower value found in (1.3); in particular (1.5) implies, again by Morse index comparison, that the ground state (positive) solution of the Hénon problem, which has Morse index 1, is not radial for $p \in\left[\bar{p}, p_{\alpha}\right)$. Indeed for the positive (i.e. $m=1$ ) radial solution $u_{p}$ (1.5) gives

$$
\mathrm{m}\left(u_{p}\right) \geq 1+N(>1)
$$

(see also [35, where the same conclusion is derived via energy comparison).

Formulas (1.4) and (1.5) have been derived both by the study of an auxiliary singular eigenvalue problem associated to the linearized operator $L_{u_{p}}$ which, in the radial setting, can be decomposed into a radial and an angular part. In particular, the study of the radial part strongly depends on the qualitative properties of the solution $u_{p}$, and the proofs of both the formulas specifically exploit the knowledge of the asymptotic behavior of $u_{p}$ as $p \rightarrow p_{\alpha}$ from the left.

In dimension $N \geq 3$ this behavior is indeed well known: all the radial solutions of (1.1) blow-up at the origin as $p \rightarrow p_{\alpha}$ and vanish elsewhere, moreover each radial solution with $m$ nodal zones is a tower of $m$ bubbles, i.e., in short, it looks like $m$ superpositions of the same limit profile

$$
U_{\alpha}(x)=\left(1+\frac{|x|^{2+\alpha}}{(N+\alpha)(N-2)}\right)^{-\frac{N-2}{2+\alpha}},
$$

with alternate sign and scaled with different speeds (for $\alpha=0$ see for instance [9, 17, 26, for $\alpha>0$ see [5, 6]). Observe that $U_{\alpha}$ is a solution of the critical equation

$$
-\Delta U_{\alpha}=|x|^{\alpha} U_{\alpha}^{p_{\alpha}}, \quad x \in \mathbb{R}^{N} .
$$

In this paper we focus on the 2-dimensional case and derive the analogous of formulas (1.4) and (1.5).

In dimension $N=2$ the asymptotic behavior of the radial solutions of (1.1) as $p \rightarrow+\infty$ (in this case the exponent $p_{\alpha}$ is substituted with $+\infty$ ) is different: one can show that all these solutions do not blow-up but concentrate at the origin and vanish elsewhere. Moreover, since $p_{\alpha}=+\infty$, the bubbling behavior is more delicate to be described and indeed profiles different than the solutions of (1.7) are involved, as shown in [25, 7] for the solution with $m=2$ nodal regions.

Very recently in [29] the results in [25, 7] have been extended to all the radial solutions of (1.1), showing that the radial solution $u_{p}$ with $m$ nodal zones (for any $m \geq 1$ ) develops a tower of $m$ bubbles, one in each nodal zone, similarly as in dimension $N \geq 3$ but, unlike the higher dimensional case, the profile of each bubble is now different, and given by

$$
Z_{\alpha, i}(x)=\log \frac{2 \theta_{i}^{2} \gamma_{i}|x|^{\frac{(\alpha+2)}{2}\left(\theta_{i}-2\right)}}{\left(\gamma_{i}+|x|^{\frac{(\alpha+2)}{2} \theta_{i}}\right)^{2}}, \quad \text { with } \quad \gamma_{i}=\frac{\theta_{i}+2}{\theta_{i}-2}\left(\frac{\theta_{i}^{2}-4}{2}\right)^{\frac{\theta_{i}}{2}}
$$

for $i=0, \ldots, m-1$, where the sequence $\left(\theta_{i}\right)_{i \in \mathbb{N}}$ is uniquely determined by the following iteration

$$
\left\{\begin{array}{l}
\theta_{0}=2 \\
\theta_{i}=\frac{2}{\mathcal{L}\left[\frac{2}{2+\theta_{i-1}} e^{-\frac{2}{2+\theta_{i-1}}}\right]}+2 \quad \text { for } i \geq 1
\end{array}\right.
$$

$\left(\mathcal{L}\right.$ is the Lambert function) and $Z_{\alpha, i}$ is a radial solution of the singular Liouville equation

$$
-\Delta Z_{\alpha, i}=\left(\frac{\alpha+2}{2}\right)^{2}|x|^{\alpha} e^{Z_{\alpha, i}}+(\alpha+2) \pi\left(2-\theta_{i}\right) \delta_{0} \quad \text { in } \mathbb{R}^{2},
$$

see Section 2 for more details. 
As a consequence of this sharp asymptotic analysis one expects that in dimension $N=2$ formulas (1.4) and (1.5) do not hold, and that the constants $\theta_{i}$ 's must be involved in the Morse index computations, for large values of $p$.

Indeed this is exactly what has been observed in the case of the radial solution $u_{p}$ with $m=2$ nodal zones, whose Morse index has been computed in 16 for the Lane-Emden problem $(\alpha=0)$ and in [7] for the Hénon problem $(\alpha>0)$. The results in [16, 7] may be summarized as follows

$$
\mathrm{m}\left(u_{p}\right) \begin{cases}=2+2\left\lceil\frac{\alpha}{2}\right\rceil+2\left\lceil\frac{2+\alpha}{4} \theta_{1}\right] & \text { if } \frac{2+\alpha}{4} \theta_{1} \notin \mathbb{N} \\ \in\left\lceil 2\left\lceil\frac{\alpha}{2}\right\rceil+\frac{2+\alpha}{2} \theta_{1}, 2+2\left\lceil\frac{\alpha}{2}\right\rceil+\frac{2+\alpha}{2} \theta_{1}\right] & \text { otherwise }\end{cases}
$$

for $p \geq \bar{p}(\alpha)(>1)$.

So far in dimension $N=2$ the value of the Morse index for all the radial solutions $u_{p}$ of (1.1) with any number $m>2$ of nodal zones, for $p$ large, was unknown. Here we fill in this gap showing that

Theorem 1.1. Let $N=2, \alpha \geq 0$ and let $u_{p}$ be a radial solution to (1.1) with $m$ nodal zones. Let $\left(\theta_{i}\right)_{i \in \mathbb{N}}$ be the sequence in (1.9). Then there exists $\bar{p}=\bar{p}(m, \alpha)>1$ such that for $p \geq \bar{p}$

$$
m\left(u_{p}\right)=m+2\left\lceil\frac{\alpha}{2}\right\rceil+2 \sum_{i=1}^{m-1}\left[\frac{2+\alpha}{4} \theta_{i}\right]
$$

if $\frac{2+\alpha}{4} \theta_{i} \notin \mathbb{N}$, for every $i=1, \ldots, m-1$. Otherwise, if $\frac{2+\alpha}{4} \theta_{i} \in \mathbb{N}$ for some index $i$, then

$$
m\left(u_{p}\right)-\left(m+2\left\lceil\frac{\alpha}{2}\right\rceil+2 \sum_{i=1}^{m-1}\left[\frac{2+\alpha}{4} \theta_{i}\right]\right) \in\left[-2 \#\left\{i=1, \ldots m-1 \mid \frac{2+\alpha}{4} \theta_{i} \in \mathbb{N}\right\}, 0\right],
$$

where [.] is the integer part and $\lceil\cdot\rceil$ the ceiling function. In particular when $\alpha=0$ (1.12) holds and it reduces to

$$
m\left(u_{p}\right)=4 m^{2}-m-2, \quad \forall p \geq \bar{p} .
$$

When $m=2$ Theorem 1.1 gives back (1.11).

Observe that, since $\theta_{i}>2$ for every $i \geq 1$ (see (1.9)), it follows that each value given by (1.12) is strictly higher than the corresponding value in the higher dimensional case given in formulas (1.4)-(1.5), and hence also higher than the Morse index lower bound in (1.3). We stress that in dimension 2, and for $\alpha>0$, the bound (1.3) has been recently improved in $[15$, by exploiting the monotonicity of the Morse index with respect to the parameter $\alpha$. It is not difficult to check that for $\alpha>0$ the value in (1.12) is in general also strictly higher than the corresponding value obtained in [15] (see Remark 6.1).

Formula (1.12) exhibits two kinds of discontinuity w.r.t. the parameter $\alpha$ : one, occurring when $\alpha$ is an even integer, is a common phenomenon also with the higher dimensional case ([6]); the other, occurring along the sequences $\alpha_{i, n}=4 n / \theta_{i}-2$, is instead peculiar of dimension 2 .

The interest in Theorem [1.1 is not just theoretical: the exact knowledge of the Morse index can be used in order to get multiplicity results for (1.1), thus clarifying the structure of the set of its solutions. 
This can be obtained for instance both via nonradial bifurcation from radial solutions associated to a change in the Morse index, and via minimization procedures in suitable symmetric settings combined with Morse index comparisons. These approaches have been explored in dimension $N \geq 3$ for all the radial solutions, while in dimension $N=2$ only the case of the radial solution with $m=2$ nodal zones has been investigated so far (see [23, 7, 4, 2]). Nevertheless there are numerical evidences that similar phenomena hold also when considering radial solutions with more than 2 nodal zones in dimension $N=2$ (see [19]), and in a subsequent paper we plan to exploit the results in Theorem 1.1 to treat this case.

The proof of Theorem 1.1 follows a similar strategy to the one developed to get (1.11) and (1.4)-(1.5): thanks to the change of variable (1.2) we can reduce to consider the Lane-Emden case $(\alpha=0)$; then, after a spectral decomposition of an auxiliary singular eigenvalue problem associated to the linearized operator, we are finally lead to study the negative eigenvalues $\nu$ of the following radial singular problem

$$
\begin{cases}-\left(r \psi^{\prime}\right)^{\prime}=r\left(p\left|u_{p}\right|^{p-1}+\frac{\nu}{r^{2}}\right) \psi & \text { as } 0<r<1, \\ \psi=0 & \text { if } r=1,\end{cases}
$$

where $u_{p}$ is the radial solution of (1.1) (with $\alpha=0$ ) with $m$ nodal zones (see Section 3 for more details). It is possible to show that negative eigenvalues for problem (1.15) may be defined and are simple ([21]), moreover they are exactly $m$ which we denote by $\nu_{j}$, $j=1, \ldots, m$. The eigenvalues $\nu_{j}$ (and eigenfunctions $\psi_{j}$ ) of (1.15) obviously depend on $u_{p}$, the core of the proof of Theorem 1.1 is thus the investigation of their asymptotic behavior as $p \rightarrow+\infty$. We prove that

Theorem 1.2. For any $j=1, \ldots, m$

$$
\lim _{p \rightarrow+\infty} \nu_{j}(p)=-\left(\frac{\theta_{m-j}}{2}\right)^{2}
$$

where $\left(\theta_{i}\right)_{i \in \mathbb{N}}$ is the sequence in (1.9).

Theorem 1.2 is part of a more general result which describes also the asymptotic behavior of the eigenfunctions (see Theorem 4.2 for the complete statement). Its proof is quite technical and, as already mentioned, it strongly relies on the tower of bubbles asymptotic behavior of the radial solution $u_{p}$ as $p \rightarrow+\infty$ described very recently in [29], for any fixed number $m \geq 1$ of nodal zones (see Section 4, see also [25, 7] for the case $m=2$ ).

The main difficulty, which is peculiar of the two dimensional case, is to understand the interaction between the different bubbles composing the profile of $u_{p}$ and the eigenfunctions of (1.15).

We shall see that each eigenfunction $\psi_{j}$ is synchronized with a different bubble: precisely the first eigenfunction $\psi_{1}$ matches with the more external nodal zone of $u_{p}$ where the last bubble $Z_{0, m-1}$ appears, the second eigenfunction $\psi_{2}$ matches with the penultimate bubble $Z_{0, m-2}$ and so on, till the last eigenfunction $\psi_{m}$ that matches with the first bubble $Z_{0,0}$ (see Section 4.1).

Indeed, in the case $\alpha=0$, one can decompose formula (1.12) as follows

$$
\mathrm{m}\left(u_{p}\right)=\sum_{i=1}^{m-1}\left(1+2\left[\frac{\theta_{m-i}}{2}\right]\right)+1,
$$

where each term " $1+2\left[\frac{\theta_{m-i}}{2}\right]$ ", coming from the $i^{\text {th }}$ eigenvalue of (1.15), describes the contribution to the Morse index due to the bubble $Z_{0, m-i}$, and the last term " 1 ", coming from the $m^{t h}$ eigenvalue, is due to the first bubble $Z_{0,0}$. Observe that the Morse index of each bubble (as a solution to (1.10) for $\alpha=0$ ) is known (see [14]) and coincides with the 
previous values:

$$
\mathrm{m}\left(Z_{0,0}\right)=1 \quad \text { and } \quad \mathrm{m}\left(Z_{0, m-i}\right)=1+2\left[\frac{\theta_{m-i}}{2}\right],
$$

so that (1.17) may be rewritten as

$$
\mathrm{m}\left(u_{p}\right)=\sum_{i=1}^{m} \mathrm{~m}\left(Z_{0, m-i}\right) .
$$

Moreover, one can explicitly compute (cfr. [29]) the different contribution coming from each bubble

$$
\mathrm{m}\left(Z_{0, m-i}\right)=1+2\left[\frac{\theta_{m-i}}{2}\right]=8(m-i)+3
$$

from which formula (1.14) follows, which is nonlinear (quadratic) in the number $m$ of nodal zones. We stress that in dimension $N \geq 3$ and for $\alpha=0$ formula (1.4) holds, which is instead linear in $m$. We notice that, since in this case the profile of the bubbles is given always by the same function $U_{0}$ (in (1.6) with $\alpha=0$ ) and it is known that $\mathrm{m}\left(U_{0}\right)=1$, formula (1.4) may be read as

$$
\mathrm{m}\left(u_{p}\right)=m+(m-1) N=\mathrm{m}\left(U_{0}\right)+(N+1) \sum_{i=1}^{m-1} \mathrm{~m}\left(U_{0}\right) .
$$

The paper is organized as follows:

\section{Contents}

1. Motivations and main results

2. Asymptotic results for the Lane-Emden problem

3. Strategy for the Morse index computation

4. Asymptotic behavior of $\nu_{j}(p)$ as $p \rightarrow+\infty$

5. The proof of Theorem 1.1 in the case $\alpha=0$

6. The proof of Theorem 1.1 in the case $\alpha>0$

7. Further results

References

\section{Asymptotic Results For the LANe-EMden PRoblem}

This section collects known results about the asymptotic behavior of the radial solutions in the case $\alpha=0$. Hence we consider the Dirichlet Lane-Emden problem

$$
\begin{cases}-\Delta u=|u|^{p-1} u & \text { in } B \\ u=0 & \text { on } \partial B\end{cases}
$$

where $p>1$ and $B$ stands for the unit disk.

For any $p>1$ and any $m \in \mathbb{N}, m \geq 1$, there exists a unique (up to a sign) radial solution to (2.1) with exactly $m-1$ interior zeros (see for instance [30, p. 263]).

The solutions do not vanish in the origin and we denote by $u_{p}$ the unique nodal radial solution of (2.1) having $m-1$ interior zeros and satisfying

$$
u_{p}(0)>0 \text {. }
$$


With a slight abuse of notation, we often write $u_{p}(r)=u_{p}(|x|)$.

2.1. Asymptotic analysis of radial solutions. Let us denote by $r_{i, p}$ the nodal radii of $u_{p}$ and by $s_{i, p}$ the critical radii of $u_{p}$ respectively, then it is known that

$$
0=s_{0, p}<r_{1, p}<s_{1, p}<r_{2, p}<\ldots<r_{m-1, p}<s_{m-1, p}<r_{m, p}=1 .
$$

Let us define the scaling parameters

$$
\varepsilon_{i, p}=\left(p\left|u_{p}\left(s_{i, p}\right)\right|^{p-1}\right)^{-\frac{1}{2}}, \quad i=0, \ldots, m-1,
$$

and rescale the solutions in each nodal zone as

$$
u_{p}^{i}(r):=p \frac{u_{p}\left(\varepsilon_{i, p} x\right)-u_{p}\left(s_{i, p}\right)}{u_{p}\left(s_{i, p}\right)} \quad \text { as } r \in\left\{\begin{array}{lc}
{\left[0, \frac{r_{1, p}}{\varepsilon_{0, p}}\right],} & \text { if } i=0, \\
{\left[\frac{r_{i, p}}{\varepsilon_{i, p}}, \frac{r_{i+1, p}}{\varepsilon_{i, p}}\right],} & \text { if } i=1, \ldots, m-1 .
\end{array}\right.
$$

Let $\left(\theta_{i}\right)_{i}$ be the sequence defined in (1.9), which satisfies (see 29]):

$$
\theta_{0}=2, \quad 8 i+2<\theta_{i}<8 i+4, \forall i \geq 1 \text {. }
$$

We also introduce

$$
Z_{i}(x):=\log \frac{2 \theta_{i}^{2} \gamma_{i}|x|^{\left(\theta_{i}-2\right)}}{\left(\gamma_{i}+|x|^{\theta_{i}}\right)^{2}}, \quad \text { where } \quad \gamma_{i}:=\frac{\theta_{i}+2}{\theta_{i}-2}\left(\frac{\theta_{i}^{2}-4}{2}\right)^{\frac{\theta_{i}}{2}} .
$$

Observe that the function $Z_{i}$ is a radial solution of

$$
\left\{\begin{array}{l}
-\Delta Z_{i}=e^{Z_{i}}+2 \pi\left(2-\theta_{i}\right) \delta_{0} \quad \text { in } \mathbb{R}^{2}, \\
Z_{i}\left(\sqrt{\frac{\theta_{i}^{2}-4}{2}}\right)=0 \\
\int_{\mathbb{R}^{2}} e^{Z_{i}} d x=\frac{8 \pi \theta_{i}}{2}
\end{array}\right.
$$

where $\delta_{0}$ is the Dirac measure centered at 0 . In particular in the case $i=0$, since the constant $\theta_{0}=2, Z_{0}$ solves the standard Liouville equation

$$
\left\{\begin{array}{l}
-\Delta Z_{0}=e^{Z_{0}} \quad x \in \mathbb{R}^{2}, \\
Z_{0}(0)=0 \\
\int_{\mathbb{R}^{2}} e^{Z_{0}} d x=8 \pi .
\end{array}\right.
$$

From [29, Theorem 2.5] we know that $u_{p}$ has a tower of bubbles behavior in the limit as $p \rightarrow+\infty$, with bubbles given by the functions $Z_{i}, i=0, \ldots, m-1$ :

Lemma 2.1 ([29]). As $p \rightarrow \infty$ we have

$$
\frac{r_{i, p}}{\varepsilon_{i, p}} \rightarrow 0(i \neq 0), \quad \frac{r_{i+1, p}}{\varepsilon_{i, p}} \rightarrow \infty, \quad \frac{s_{i, p}}{\varepsilon_{i, p}} \rightarrow \sqrt{\frac{\theta_{i}^{2}-4}{2}},
$$

for $i=0, \ldots m-1$. Furthermore

$$
\begin{aligned}
& u_{p}^{0} \longrightarrow Z_{0} \text { in } C_{\mathrm{loc}}^{1}\left(\mathbb{R}^{2}\right), \\
& u_{p}^{i} \longrightarrow Z_{i} \text { in } C_{\mathrm{loc}}^{1}\left(\mathbb{R}^{2} \backslash\{0\}\right), \text { for } i=1, \ldots m-1 .
\end{aligned}
$$

Last we recall some pointwise estimates that will be useful in the study of the linearized operator at $u_{p}$. Let $f_{p}$ be the following function

$$
f_{p}(r):=p r^{2}\left|u_{p}(r)\right|^{p-1}, \quad 0 \leq r \leq 1
$$

and for any $K>1$ and $p>1$ let us define the set $G_{p}(K) \subset[0,1]$ as

$$
G_{p}(K):=\bigcup_{i=0}^{m-2}\left[K \varepsilon_{i, p}, \frac{1}{K} \varepsilon_{i+1, p}\right] \cup\left[K \varepsilon_{m-1, p}, 1\right] .
$$

In [16, Proposition 6.10] it has been proven that 
Lemma 2.2. There exists $C>0$ such that

$$
f_{p}(r) \leq C \text { for any } r \geq 0 \text { and } p>1 .
$$

Moreover for any $\delta>0$ there exist $K(\delta)>1$ and $p(\delta)>1$ such that for any $K>K(\delta)$ and $p \geq p(\delta)$

$$
\max \left\{f_{p}(r): r \in G_{p}(K)\right\} \leq \delta .
$$

\section{Strategy for the Morse index Computation}

We will first consider the Lane-Emden problem $(\alpha=0)$ and prove Theorem 1.1 in this case (see Section 5), finally in Section 6 we will treat the Hénon problem $(\alpha>0)$ by exploiting the change of variable (1.2) and prove Theorem 1.1 in its full generality.

This section describes the strategy that we will adopt in order to compute the Morse index in the case $\alpha=0$. More precisely we will show how the computation of the Morse index may be reduced to the study of the size of the negative radial eigenvalues of a suitable singular eigenvalue problem (see formula (3.13) below). The study of these eigenvalues and the conclusion of the proof of Theorem 1.1 (in the case $\alpha=0$ ) is instead the goal of Sections 4 and 5, respectively.

As before we denote by $u_{p}$ the radial solution to the Lane-Emden problem (2.1) having $m-1$ interior zeros and keep all the notations introduced in Section 2.

As already recalled the Morse index of $u_{p}$ is the maximal dimension of a subspace of $H_{0}^{1}(B)$ in which the quadratic form

$$
\mathcal{Q}_{p}(\phi)=\int_{B}\left(|\nabla \phi|^{2}-V_{p}(x) \phi^{2}\right) d x
$$

is negative defined, where

$$
V_{p}(x):=p\left|u_{p}(x)\right|^{p-1} .
$$

Since $u_{p}$ is a radial solution we can also consider the radial Morse index of $u_{p}$, denoted by $\mathrm{m}_{\text {rad }}\left(u_{p}\right)$, which is the maximal dimension of a subspace $X$ of $H_{0 \text {,rad }}^{1}(B)$ (the subspace of radial functions in $\left.H_{0}^{1}(B)\right)$ such that $\mathcal{Q}_{p}(\phi)<0, \forall \phi \in X \backslash\{0\}$.

Observe that $B$ is a bounded domain, so $\mathrm{m}\left(u_{p}\right)$ (resp. $\left.\mathrm{m}_{\mathrm{rad}}\left(u_{p}\right)\right)$ coincides with the number of the negative eigenvalues (resp. radial eigenvalues) $\Lambda(p)$, counted with multiplicity, of the linearized operator $L_{p}:-\Delta-V_{p}(x)$ at $u_{p}$, i.e.:

$$
-\Delta \phi-V_{p}(x) \phi=\Lambda(p) \phi, \quad \phi \in H_{0}^{1}(B)\left(\text { resp. } \phi \in H_{0, \mathrm{rad}}^{1}(B)\right) .
$$

It is well known (see [28, 11]) that

$$
\mathrm{m}_{\mathrm{rad}}\left(u_{p}\right)=m,
$$

where $m$ is the number of nodal zones of $u_{p}$, moreover $u_{p}$ is radially non-degenerate (see for instance 23]).

In order to computer $\mathrm{m}\left(u_{p}\right)$ we follow the same general strategy already used in [17, 16, 6. 7, 23]: instead of counting the negative eigenvalues of (3.3), we consider an auxiliary singular eigenvalue problem which allow to exploit a spectral decomposition and hence to reduce to a radial eigenvalue problem. 


\subsection{Singular eigenvalue problem and spectral decomposition.}

It is possible to show that $\mathrm{m}\left(u_{p}\right)$ coincides with the number of negative eigenvalues $\widehat{\Lambda}(p)$, counted with multiplicity, of the following auxiliary eigenvalue problem associated to the linearized operator $L_{p}$ :

$$
-\Delta \phi-V_{p}(x) \phi=\widehat{\Lambda}(p) \frac{\phi}{|x|^{2}}, \quad \phi \in \mathcal{H}_{0},
$$

in the weighted Sobolev space

$$
\mathcal{H}_{0}=\mathcal{L} \cap H_{0}^{1}(B), \text { where } \mathcal{L}=\left\{\phi: B \rightarrow \mathbb{R}: \phi /|x| \in L^{2}(B)\right\} .
$$

This equivalence is quite straightforward in the case of domains which do not contain the origin (see for instance [22, where it is proved in the case when the domain is an annulus). In our case, since $0 \in B,(3.5)$ is a singular problem. Nevertheless its negative eigenvalues may be variationally characterized despite a lack of compactness (see 21, for more details see also [23, Section 3.2], and [3] for a more general setting) and the equivalence between the number of the negative eigenvalues of (3.3) and (3.5) can be proved (see [21, Lemma 2.6], see also [23, Lemma 3.5], [3, Proposition 1.1]).

The main advantage of dealing with the singular problem (3.5) instead of (3.3) is that the eigenfunctions of (3.5) can be easily projected along the spherical harmonics. This implies a spectral decomposition for the eigenvalues $\widehat{\Lambda}(p)$ of (3.5) into a radial and an angular part:

$$
\widehat{\Lambda}(p)=k^{2}+\nu(p),
$$

where $k^{2}$, for $k=0,1,2, \ldots$ are the eigenvalues of the Laplace-Beltrami operator $-\Delta_{\mathbb{S}^{1}}$ (the angular part) and $\nu(p)$ are the (negative) radial eigenvalues of (3.5), namely they satisfy the following singular Sturm-Liouville problem

$$
-\left(r \psi^{\prime}\right)^{\prime}=r\left(V_{p}(r)+\frac{\nu(p)}{r^{2}}\right) \psi, \quad \psi \in \mathcal{H}_{0, \mathrm{rad}}=\mathcal{L} \cap H_{0, \mathrm{rad}}^{1}(B) .
$$

We stress that $\widehat{\Lambda}(p)$ is negative iff

$$
\sqrt{-\nu(p)}>k \text {. }
$$

Hence in order to compute $\mathrm{m}\left(u_{p}\right)$ one reduces to study (3.8) for the negative eigenvalues $\nu(p)$ of the 1-dimensional problem (3.7).

For more details about the spectral decomposition the reader may look at $32,22,21$, or to the more recent [23, Lemma 3.7], 3, Section 4].

\subsection{Variational characterization of the negative eigenvalues and eigenfunctions of (3.7).}

As already said, the negative eigenvalues for problem (3.7) may be defined variationally despite the singularity of the Sturm-Liouville problem (3.7) at the origin, moreover they are simple and by (3.4) we know that they are exactly $m$, which we denote by $\nu_{j}(p), j=1, \ldots, m$. Here we recall their variational characterization and the definition of the corresponding eigenfunctions (cfr. 21], see also [3, Section 3]):

$$
\nu_{1}(p):=\min \left\{\frac{\int_{0}^{1} r\left(\left|\psi^{\prime}\right|^{2}-V_{p} \psi^{2}\right) d r}{\int_{0}^{1} r^{-1} \psi^{2} d r}: \psi \in \mathcal{H}_{0, \mathrm{rad}}, \psi \neq 0\right\} ;
$$

since it is negative, it can be proven that it is attained by a function $\psi_{1, p} \in \mathcal{H}_{0, \text { rad }}$ which solves (3.7) in a weak sense, and which is therefore called an eigenfunction related to the 
eigenvalue $\nu_{1}(p)$; w.l.g. we may assume that it is normalized in $\mathcal{L}$, i.e. $\int_{0}^{1} r^{-1}\left(\psi_{1, p}\right)^{2}=1$. Iteratively, for $j=2, \ldots, m$, one has

$$
\nu_{j}(p):=\min \left\{\frac{\int_{0}^{1} r\left(\left|\psi^{\prime}\right|^{2}-V_{p} \psi^{2}\right) d r}{\int_{0}^{1} r^{-1} \psi^{2} d r}: \psi \in \mathcal{H}_{0, \mathrm{rad}}, \psi \perp \psi_{1, p}, \ldots \psi_{j-1, p}\right\},
$$

where the symbol $\perp$ denotes orthogonality in $\mathcal{L}$, i.e.

$$
\varphi \perp \psi \Longleftrightarrow \int_{0}^{1} r^{-1} \varphi \psi d r=0,
$$

Again, since $\nu_{j}(p)<0$ for any $j=2, \ldots, m$, then the infimum is attained by an eigenfunction $\psi_{j, p}$, which solves (3.7) in a weak sense and that w.l.g. satisfies

$$
\int_{0}^{1} r^{-1} \psi_{j, p} \psi_{h, p} d r=\delta_{j h}
$$

Furthermore one can prove that the eigenvalues are simple and that (see 8 , Proposition 3.3 , Theorem 1.3])

$$
\nu_{1}(p)<\nu_{2}(p)<\ldots \nu_{m-1}(p)<-1<\nu_{m}(p)<0,
$$

for any $p>1$.

\subsection{Computation of $\mathbf{m}\left(u_{p}\right)$ by the size of the negative eigenvalues of (3.7).}

By (3.6) and (3.8), and recalling that the eigenvalues $\nu_{j}(p)$ defined in (3.9)-(3.10) are simple while the eigenvalues $k^{2}$ of the Laplace-Beltrami operator $-\Delta_{\mathbb{S} 1}$ have multiplicity 1 if $k=0$ and 2 when $k \geq 1$, it follows that

$$
\mathrm{m}\left(u_{p}\right)=m+2 \sum_{j=1}^{m-1}\left\lceil\sqrt{-\nu_{j}(p)}-1\right\rceil
$$

for any $p>1$.

\section{Asymptotic Behavior of $\nu_{j}(p)$ as $p \rightarrow+\infty$}

In this section we study the asymptotic behavior, as $p \rightarrow+\infty$, of the singular eigenvalues $\nu_{j}(p), j=1, \ldots, m$, defined in (3.9) $-(3.10)$.

In order to compute their limit values we will properly scale the corresponding eigenfunctions $\psi_{j, p}$ according to each scaling parameter $\varepsilon_{i, p}$ introduced in (2.2) and then pass to the limit into the equations satisfied by the rescaled functions. This will be possible thanks to the asymptotic results on the solutions $u_{p}$ of the Lane-Emden problem (2.1) collected in Section 2 Furthermore we will analyze the limit eigenvalue problems obtained (see Lemma 4.1 below).

Our results about the asymptotic behavior of the eigenvalues and the rescaled eigenfunctions are stated in Theorem 4.2 below (which is the complete version of Theorem 1.2 in Section 1).

Next we introduce some notation and observations needed to state Theorem 4.2 . 
We denote by $\psi_{j, p}^{i}$, for $i=0, \ldots m-1$, the $m$ functions obtained from rescaling each eigenfunction $\psi_{j, p}$ as follows:

$$
\psi_{j, p}^{i}(r):= \begin{cases}\psi_{j, p}\left(\varepsilon_{i, p} r\right) & \text { in }\left[0, \frac{1}{\varepsilon_{i, p}}\right) \\ 0 & \text { otherwise. }\end{cases}
$$

Observe that $\psi_{j, p}^{i}$ belong to the closure of $C_{0}^{\infty}(0, \infty)$ with respect to the norm

$$
\left(\int_{0}^{\infty}\left(r\left|\psi^{\prime}\right|^{2}+r^{-1} \psi^{2}\right) d r\right)^{\frac{1}{2}}
$$

which will be denoted by $\mathcal{D}_{\text {rad }}$, and solve

$$
-\left(r\left(\psi_{j, p}^{i}\right)^{\prime}\right)^{\prime}=r\left(V_{p}^{i}+\frac{\nu_{j}(p)}{r^{2}}\right) \psi_{j, p}^{i}
$$

in $\left[0, \frac{r_{1, p}}{\varepsilon_{0, p}}\right]$ if $i=0$, in $\left[\frac{r_{i, p}}{\varepsilon_{i, p}}, \frac{r_{i+1, p}}{\varepsilon_{i, p}}\right]$ if $i=1, \ldots, m-1$, with

$$
V_{p}^{i}(r):=\left(\varepsilon_{i, p}\right)^{2} V_{p}\left(\varepsilon_{i, p} r\right)
$$

where $V_{p}$ is defined in (3.2). Moreover by the definition (4.1) and the normalization (3.11), we have

$$
\begin{gathered}
\int_{0}^{\infty} r^{-1}\left(\psi_{j, p}^{i}\right)^{2} d r \leq \int_{0}^{1} r^{-1}\left(\psi_{j, p}\right)^{2} d r=1 \\
\int_{0}^{\infty} r\left(\left(\psi_{j, p}^{i}\right)^{\prime}\right)^{2} d r \leq \int_{0}^{1} r\left(\psi_{j, p}^{\prime}\right)^{2} d r .
\end{gathered}
$$

Thanks to Lemma 2.1 the set $\left[0, \frac{r_{1, p}}{\varepsilon_{0, p}}\right)$ invades $[0, \infty)$ in the limit as $p \rightarrow+\infty$, while the sets $\left(\frac{r_{i, p}}{\varepsilon_{i, p}}, \frac{r_{i+1, p}}{\varepsilon_{i, p}}\right)$, for $i=1, \ldots m-1$, invade $(0, \infty)$. Furthermore

$$
\begin{aligned}
V_{p}^{0} & =\left(1+\frac{u_{p}^{0}}{p}\right)^{p-1} \longrightarrow e^{Z_{0}} \text { in } C_{\mathrm{loc}}^{0}[0, \infty), \\
V_{p}^{i} & =\left(1+\frac{u_{p}^{i}}{p}\right)^{p-1} \longrightarrow e^{Z_{i}} \text { in } C_{\mathrm{loc}}^{0}(0, \infty), \text { for } i=1, \ldots, m-1,
\end{aligned}
$$

where $Z_{i}$ are the functions in (2.5). Hence, if we prove that we can pass to the limit into equations (4.2), then the natural limit problems will be the following eigenvalue problems

$$
\left\{\begin{array}{l}
-\left(r(\eta)^{\prime}\right)^{\prime}=r\left(e^{Z^{i}}+\frac{\beta}{r^{2}}\right) \eta \quad \text { as } r>0 \\
\eta \in \mathcal{D}_{\mathrm{rad}}
\end{array}\right.
$$

for $i=0, \ldots, m-1$. From [17, Section 5] and [7, Section 5.2] we know that (4.8) admits only one negative eigenvalue, which can be explicitly characterized:

Lemma 4.1. Let $i \in\{0, \ldots, m-1\}$ and let $\beta$ be an eigenvalue to (4.8). Then

$$
\beta<0 \quad \text { iff } \quad \beta=\beta^{i}:=-\left(\frac{\theta_{i}}{2}\right)^{2},
$$

where $\theta_{i}$ is the number given by (1.9). Moreover in such a case the eigenvalue $\beta^{i}$ is simple and its eigenspace is spanned by

$$
\eta(r)=\eta^{i}(r):=\frac{\sqrt{\theta_{i} \gamma_{i}} r^{\frac{\theta_{i}}{2}}}{\gamma_{i}+r^{\theta_{i}}}, \quad \text { where } \quad \gamma_{i}:=\frac{\theta_{i}+2}{\theta_{i}-2}\left(\frac{\theta_{i}^{2}-4}{2}\right)^{\frac{\theta_{i}}{2}}
$$


Notice that $\eta^{i}$ is normalized so that

$$
\int_{0}^{\infty} r^{-1}\left(\eta^{i}\right)^{2} d r=1
$$

As a consequence of Lemma 4.1 it follows that all the numbers $\beta=\beta^{i}$ in (4.9), for $i=0, \ldots, m-1$, are candidates to be the limit value of each eigenvalue $\nu_{j}(p)$, as $p \rightarrow+\infty$.

We remark that, for $i=0, \ldots, m-1$, the limit problems (4.8), as well as their negative eigenvalue $\beta^{i}$ in (4.9), are different from one another, in particular combining (4.9) and (2.4) we know that the following strict order holds:

$$
\beta^{m-1}<\ldots \beta^{1}<-25<\beta^{0}=-1 .
$$

In order to select the right limit value of $\nu_{j}(p)$ among all the $\beta^{i}$ 's, we need thus to understand which one (if any) among the possible scalings $\psi_{j, p}^{i}$, for $i=0, \ldots, m-1$, does not vanish as $p \rightarrow \infty$.

We shall see that

Theorem 4.2. For any $j=1, \ldots, m$

$$
\lim _{p \rightarrow+\infty} \nu_{j}(p)=\beta^{m-j}=-\left(\frac{\theta_{m-j}}{2}\right)^{2}
$$

Moreover there exists $A_{j} \neq 0$ such that

$$
\begin{aligned}
& \psi_{j, p}^{m-j} \rightarrow A_{j} \eta^{m-j} \\
& \psi_{j, p}^{i} \rightarrow 0, \quad i=0, \ldots, m-1, i \neq m-j
\end{aligned}
$$

weakly in $\mathcal{D}_{\text {rad }}$ and strongly in $C_{\text {loc }}^{1}(0, \infty)$.

Observe that Theorem 4.2 describes the asymptotic also for the last eigenvalue $\nu_{m}(p)$, even if this is not needed for the computation of the Morse index.

4.1. The proof of Theorem 4.2, The proof of Theorem 4.2 is based on an iterative procedure on the index $j$.

First we prove the result for $j=1$ :

Proposition 4.3.

$$
\lim _{p \rightarrow+\infty} \nu_{1}(p)=\beta^{m-1}
$$

Moreover there exists $A_{1} \neq 0$ such that

$$
\begin{aligned}
& \psi_{1}^{m-1} \rightarrow A_{1} \eta^{m-1} \\
& \psi_{1}^{i} \rightarrow 0, \quad i=0, \ldots, m-2
\end{aligned}
$$

Then we prove the inductive step

Proposition 4.4. Let $h \in\{2, \ldots, m-1\}$. Assume that Theorem 4.2 holds true for any $j=1, \ldots, h-1$. Then it holds true for $j=h$.

The last eigenvalue has to be treated separately, namely we conclude proving

\section{Proposition 4.5 .}

$$
\lim _{p \rightarrow+\infty} \nu_{m}(p)=\beta^{0}=-1
$$

Moreover there exists $A_{m} \neq 0$ such that

$$
\begin{aligned}
& \psi_{m}^{0} \rightarrow A_{m} \eta^{0} \\
& \psi_{m}^{i} \rightarrow 0, \quad i=1,2, \ldots, m-1 .
\end{aligned}
$$


4.2. Preliminary convergence results. We start showing that the eigenvalues $\nu_{j}(p)$ and the rescaled eigenfunctions $\psi_{j, p}^{i}$ are uniformly bounded in $p$.

Lemma 4.6. There exists $C>0$ such that for every $p>1$ we have

$$
\begin{gathered}
-C \leq \nu_{1}(p)<\nu_{2}(p)<\ldots<\nu_{m}(p)<0 \\
\int_{0}^{\infty} r\left(\left(\psi_{j, p}^{i}\right)^{\prime}\right)^{2} d r \leq C
\end{gathered}
$$

for every $i=0, \ldots, m-1$ and $j=1, \ldots, m$.

Proof. Using $\psi_{j, p}$ as a test function in (3.7) we get

$$
\int_{0}^{1} r\left(\psi_{j, p}^{\prime}\right)^{2} d r=\int_{0}^{1} r\left(V_{p}+\frac{\nu_{j}(p)}{r^{2}}\right)\left(\psi_{j, p}\right)^{2} d r
$$

For $j=1$, by virtue of (3.11) we can extract $\nu_{1}(p)$ getting that

$$
\nu_{1}(p)=\int_{0}^{1} r\left(\left(\psi_{1, p}^{\prime}\right)^{2}-p\left|u_{p}\right|^{p-1}\left(\psi_{1, p}\right)^{2}\right) d r \geq-\sup _{(0,1)} f_{p}(r) \int_{0}^{1} r^{-1}\left(\psi_{1, p}\right)^{2} d r=-C
$$

thanks to Lemma 2.2 and (4.4).

Besides, since $\nu_{j}(p)<0$ for $j=1, \ldots, m$ by (3.12), 4.18), (4.4) and Lemma 2.2

$$
\int_{0}^{1} r\left(\psi_{j, p}^{\prime}\right)^{2} d r<\int_{0}^{1} r^{-1} f_{p}\left(\psi_{j, p}\right)^{2} d r \leq \sup _{r \in(0,1)} f_{p}(r) \int_{0}^{1} r^{-1}\left(\psi_{j, p}\right)^{2} d r=C .
$$

So also (4.17) is proved, recalling (4.5).

As a consequence we can thus prove:

Proposition 4.7. Let $j=1, \ldots, m$. Then there exist a sequence $p_{n} \rightarrow+\infty$, a number $\bar{\nu}_{j} \leq 0$ and $m$ functions $\bar{\psi}_{j}^{i}$, for $i=0, \ldots, m-1$, such that as $n \rightarrow+\infty$

$$
\begin{aligned}
\nu_{j}\left(p_{n}\right) & \rightarrow \bar{\nu}_{j} \\
\psi_{j, p_{n}}^{i} & \rightarrow \bar{\psi}_{j}^{i} \quad \text { weakly in } \mathcal{D}_{\mathrm{rad}} \text { and strongly in } L_{\mathrm{loc}}^{2}(0, \infty) .
\end{aligned}
$$

Moreover $\bar{\psi}_{j}^{i}$ is a weak solution to (4.8) with eigenvalue $\beta=\bar{\nu}_{j}$.

Proof. By (4.16) we can extract a sequence $p_{n} \rightarrow+\infty$ such that $\nu_{j}\left(p_{n}\right) \rightarrow \bar{\nu}_{j} \leq 0$. (4.4) and (4.17) imply that the sequence $\left(\psi_{j, p_{n}}^{i}\right)_{n}$ is uniformly bounded in $\mathcal{D}_{\text {rad }}$ hence, up to another subsequence (that we still denote by $p_{n}$ ), one has that $\psi_{j, p_{n}}^{i} \rightarrow \bar{\psi}_{j}^{i}$ weakly in $\mathcal{D}_{\text {rad}}$, strongly

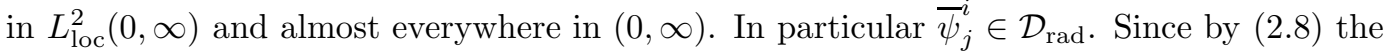
intervals

$$
I_{p}^{i}:= \begin{cases}\left(0, \frac{r_{1, p}}{\varepsilon_{0, p}}\right) & \text { if } i=0 \\ \left(\frac{r_{i, p}}{\varepsilon_{i, p}}, \frac{r_{i+1, p}}{\varepsilon_{i, p}}\right) & \text { if } i>0\end{cases}
$$

invade $(0, \infty)$, as $p \rightarrow+\infty$, for every $\varphi \in C_{0}^{\infty}(0, \infty)$ we can choose $n$ so large in such a way that $\operatorname{supp} \varphi \subset I_{p_{n}}^{i}$ and $\psi_{j, p_{n}}^{i}$ verifies

$$
\int_{0}^{\infty} r\left(\psi_{j, p_{n}}^{i}\right)^{\prime} \varphi^{\prime} d r=\int_{0}^{\infty} r V_{p_{n}}^{i} \psi_{j, p_{n}}^{i} \varphi d r+\nu_{j}\left(p_{n}\right) \int_{0}^{\infty} r^{-1}\left(\psi_{j, p_{n}}^{i}\right) \varphi d r .
$$

The weak convergence in $\mathcal{D}_{\text {rad }}$ then implies that

$$
\begin{gathered}
\int_{0}^{\infty} r\left(\psi_{j, p_{n}}^{i}\right)^{\prime} \varphi^{\prime} d r \rightarrow \int_{0}^{\infty} r\left(\bar{\psi}_{j}^{i}\right)^{\prime} \varphi^{\prime} d r \\
\int_{0}^{\infty} r \psi_{j, p_{n}}^{i} \varphi d r \rightarrow \int_{0}^{\infty} r \bar{\psi}_{j}^{i} \varphi d r
\end{gathered}
$$


while the strong convergence in $L_{\mathrm{loc}}^{2}$ and the fact that $V_{p_{n}}^{i} \rightarrow e^{Z_{i}}$ in $C_{\mathrm{loc}}^{1}(0, \infty)$ imply also that

$$
\int_{0}^{\infty} r V_{p_{n}}^{i} \psi_{j, p_{n}}^{i} \varphi d r \rightarrow \int_{0}^{\infty} r e^{Z_{i}} \bar{\psi}_{j}^{i} \varphi d r
$$

getting that $\bar{\psi}_{j}^{i}$ solves (4.8) with $\beta=\bar{\nu}_{j}$ in the weak sense.

Thanks to Lemma 4.1, we can deduce some crucial consequences of Proposition 4.7

Corollary 4.8. Let $\bar{\nu}_{j}$ and $\bar{\psi}_{j}^{i}$ be as in Proposition 4.7 and $\beta^{i}, \eta^{i}$ as in (4.9) and (4.10). It holds

(i) If $\bar{\nu}_{j} \neq \beta^{i}, 0$, then $\bar{\psi}_{j}^{i} \equiv 0$.

(ii) If there exists $j \in\{1, \ldots, m-1\}$ such that $\bar{\psi}_{j}^{i} \not \equiv 0$, then $\bar{\nu}_{j}=\beta^{i}$. Furthermore

$$
\begin{aligned}
& \bar{\psi}_{j}^{i}=A_{j} \eta^{i} \text { for some } A_{j} \neq 0,\left|A_{j}\right| \leq 1 \\
& \bar{\psi}_{j}^{h} \equiv 0 \text { for every } h \neq i .
\end{aligned}
$$

Proof. (i) is a direct consequence of Proposition 4.7 and Lemma 4.1. Indeed $\bar{\nu}_{j} \leq 0$ is an eigenvalue of problem (4.8) and the only strictly negative eigenvalue must be $\beta^{i}$.

The first assertion of (ii) follows from (i), observing also that, thanks to (3.12), $\bar{\nu}_{j} \leq-1$ as $j=1, \ldots, m-1$ (while $-1 \leq \bar{\nu}_{m} \leq 0$ ). Then Lemma 4.1 implies that $\bar{\psi}_{j}^{i}=A_{j} \eta^{i}$, for a certain $A_{j} \in \mathbb{R}$. As a consequence, by the convergence in (4.20) and Fatou's Lemma, one deduces that

$$
\left(A_{j}\right)^{2} \stackrel{\text { (4.11) }}{=}\left(A_{j}\right)^{2} \int_{0}^{\infty} r^{-1}\left(\eta^{i}\right)^{2}=\int_{0}^{\infty} r^{-1}\left(\bar{\psi}_{j}^{i}\right)^{2} \leq \liminf _{p \rightarrow+\infty} \int_{0}^{\infty} r^{-1}\left(\psi_{j, p}^{i}\right)^{\stackrel{\text { (4.44) }}{\leq}} 1,
$$

which implies (4.21). Finally $0 \neq \bar{\nu}_{j}=\beta^{i} \neq \beta^{h}$, for $h \neq i$, by (4.12), hence (4.22) follows from $(i)$.

The convergence in (4.20) is actually stronger, as stated by the following Lemma.

Lemma 4.9. Using the same notation of Proposition 4.7, we have

$$
\psi_{j, p_{n}}^{i} \rightarrow \bar{\psi}_{j}^{i} \text { strongly in } C_{\mathrm{loc}}^{1}(0, \infty),
$$

as $n \rightarrow+\infty$, for $j=1, \ldots, m, i=0, \ldots, m-1$.

Furthermore, if $\bar{\nu}_{j} \leq-25$, then

$$
\psi_{j, p_{n}}^{0} \rightarrow \bar{\psi}_{j}^{0} \text { in } C_{\mathrm{loc}}^{1}[0, \infty)
$$

as $n \rightarrow+\infty$, for $j=1, \ldots, m$.

Proof. Recall tha $\psi_{j, p_{n}} \in \mathcal{H}_{0, \text { rad }} \subset C^{0}(0,1]$ and $\psi_{j, p_{n}}$ is a solution to (3.7) (with $V_{p_{n}} \in$ $\left.C^{\infty}[0,1]\right)$, so $\psi_{j, p_{n}} \in C^{1}(0,1]$ and in turn via a bootstrap argument $\psi_{j, p_{n}} \in C^{\infty}(0,1]$. If $r_{2} \geq r_{1} \geq R^{-1}>0$ we have

$$
\left|\psi_{j, p_{n}}^{i}\left(r_{2}\right)-\psi_{j, p_{n}}^{i}\left(r_{1}\right)\right| \leq \int_{r_{1}}^{r_{2}}\left|\left(\psi_{j, p_{n}}^{i}\right)^{\prime}(t)\right| d t \stackrel{\sqrt[4.177]{\leq}}{\leq} C\left(\int_{r_{1}}^{r_{2}} t^{-1} d t\right)^{\frac{1}{2}} \leq C R^{\frac{1}{2}} \sqrt{r_{2}-r_{1}}
$$

so (up to another subsequence) $\psi_{j, p_{n}}^{i} \rightarrow \bar{\psi}_{j}^{i}$ uniformly in any set of type $\left[R^{-1}, R\right]$ by the Arzelà-Ascoli Theorem. Furthermore, by equation (4.2), it is easy to derive a bound for $\psi_{j, p_{n}}^{i}$ in $C^{2}\left(R^{-1}, R\right)$, which ensures the convergence in $C^{1}\left(R^{-1}, R\right)$, completing the proof of (4.23).

Next we derive (4.24). 
Reasoning as in [21, Lemma 2.4] or [23, Proposition 2.2] and integrating the equation (3.7) one has

$$
\psi_{j, p_{n}}(\rho)=\rho^{\kappa_{j, p_{n}}} \int_{\rho}^{1} s^{-1-2 \kappa_{j, p_{n}}} \int_{0}^{s} t^{1+\kappa_{j, p_{n}}} V_{p_{n}}(t) \psi_{j, p_{n}}(t) d t d s
$$

where $\kappa_{j, p_{n}}=\sqrt{\left|\nu_{j}\left(p_{n}\right)\right|}>4$ by assumption. Observe that

$$
\begin{aligned}
\left|\int_{0}^{s} t^{1+\kappa_{j, p_{n}}} V_{p_{n}}(t) \psi_{j, p_{n}}(t) d t\right| & \leq\left\|V_{p_{n}}\right\|_{\infty}\left|\int_{0}^{s} t^{-\frac{1}{2}} \psi_{j, p_{n}}(t) t^{\kappa_{j, p_{n}}+\frac{3}{2}} d t\right| \\
& \stackrel{\text { Hölder }}{\leq}\left\|V_{p_{n}}\right\|_{\infty}\left(\int_{0}^{1} \frac{\left(\psi_{j, p_{n}}(t)\right)^{2}}{t} d t\right)^{\frac{1}{2}}\left(\int_{0}^{s} t^{3+2 \kappa_{j, p_{n}}} d t\right)^{\frac{1}{2}} \\
& \stackrel{(\star)}{\leq} \varepsilon_{0, p}^{-2} \frac{s^{2+\kappa_{j, p_{n}}}}{\sqrt{4+2 \kappa_{j, p_{n}}}} \leq \frac{\varepsilon_{0, p}^{-2}}{2} s^{2+\kappa_{j, p_{n}}}
\end{aligned}
$$

where $(\star)$ follows from the normalization (4.4) and the fact that $\left\|V_{p_{n}}\right\|_{\infty} \leq \varepsilon_{0, p}^{-2}$ by (3.2) and (2.2). Inserting this estimate in (4.25) we get

$$
\left|\psi_{j, p_{n}}(\rho)\right| \leq \frac{\varepsilon_{0, p}^{-2}}{2} \rho^{\kappa_{j, p_{n}}} \int_{\rho}^{1} s^{1-\kappa_{j, p_{n}}} d s \leq \frac{\varepsilon_{0, p}^{-2}}{2} \rho^{\kappa_{j, p_{n}}} \frac{1-\rho^{2-\kappa_{j, p_{n}}}}{2-\kappa_{j, p_{n}}} \stackrel{\kappa_{j, p_{n}}>4}{\leq} \varepsilon_{0, p}^{-2} \rho^{2} .
$$

This implies that $\psi_{j, p_{n}}$ is continuous and differentiable in $\rho=0$ with $\psi_{j, p_{n}}(0)=\left(\psi_{j, p_{n}}\right)^{\prime}(0)=$ 0 . Then we can integrate (3.7) in $(0, \rho)$ getting

$$
\rho\left(\psi_{j, p_{n}}\right)^{\prime}(\rho)=-\int_{0}^{\rho}\left(s V_{p_{n}}(s)+\frac{\nu_{j}\left(p_{n}\right)}{s}\right) \psi_{j, p_{n}}(s) d s .
$$

Combining with 4.26 we derive

$$
\begin{aligned}
\left|\left(\psi_{j, p_{n}}\right)^{\prime}(\rho)\right| & \leq \frac{\varepsilon_{0, p}^{-2}}{\rho} \int_{0}^{\rho}\left(s\left\|V_{p_{n}}\right\|_{\infty}+\frac{\left|\nu_{j}\left(p_{n}\right)\right|}{s}\right) s^{2} d s \\
& \stackrel{(*)}{\leq} \frac{\varepsilon_{0, p}^{-2}}{\rho}\left(\varepsilon_{0, p}^{-2} \frac{\rho^{4}}{4}+C \frac{\rho^{2}}{2}\right) \\
& \leq \varepsilon_{0, p}^{-2} \rho\left(\varepsilon_{0, p}^{-2} \rho^{2}+C\right),
\end{aligned}
$$

where in $(*)$ we have used (3.2), the fact that $\left\|V_{p_{n}}\right\|_{\infty}=p u_{p_{n}}(0)^{p-1}$ (since $\left\|u_{p}\right\|_{\infty}=u_{p}(0)$, cfr. [29]), (2.2) and (4.16). This implies that $\psi_{j, p_{n}} \in C^{1}[0,1]$. Furthermore by (3.7)

$$
-\psi_{j, p_{n}}^{\prime \prime}(\rho)=\frac{\psi_{j, p_{n}}^{\prime}(\rho)}{\rho}+\left(\rho^{2} V_{p_{n}}(\rho)+\nu_{j}\left(p_{n}\right)\right) \frac{\psi_{j, p_{n}}(\rho)}{\rho^{2}}, \quad \text { for } \rho \in(0,1],
$$

so using (4.26), (4.27) and (4.16)

$$
\left|\psi_{j, p_{n}}^{\prime \prime}(\rho)\right| \leq 2 \varepsilon_{0, p_{n}}^{-2}\left(\varepsilon_{0, p_{n}}^{-2} \rho^{2}+\widetilde{C}\right) \quad \text { for } \rho \in(0,1] .
$$

By (2.8) for any $R>0$ there exists $n$ large enough such that $R<\frac{r_{1, p_{n}}}{\varepsilon_{0, p_{n}}}$. Recalling the definition of the rescaled function (4.1), by the regularity of $\psi_{j, p_{n}}$, we conclude that $\psi_{j, p_{n}}^{0} \in$ $C^{1}[0, R] \cap C^{\infty}(0, R]$. Scaling into the estimates (4.26), (4.27), (4.28) we obtain that for $r \in[0, R]:$

$$
\begin{gathered}
\left|\psi_{j, p_{n}}^{0}(r)\right|=\left|\psi_{j, p_{n}}\left(\varepsilon_{0, p_{n}} r\right)\right| \stackrel{\sqrt{4.26}}{\leq} r^{2}, \\
\left|\left(\psi_{j, p_{n}}^{0}\right)^{\prime}(r)\right|=\varepsilon_{0, p}\left|\psi_{j, p_{n}}^{\prime}\left(\varepsilon_{0, p_{n}} r\right)\right| \stackrel{\text { 4.27 }}{\leq}\left(r^{2}+C\right) r \leq C_{R} r, \\
\left|\left(\psi_{j, p_{n}}^{0}\right)^{\prime \prime}(r)\right|=\varepsilon_{0, p_{n}}^{2}\left|\psi_{j, p_{n}}^{\prime \prime}\left(\varepsilon_{0, p_{n}} r\right)\right| \stackrel{\text { (4.28) }}{\leq} 2\left(r^{2}+\widetilde{C}\right) \leq \widetilde{C}_{R} \quad, \text { for } r \in(0, R]
\end{gathered}
$$

thus $\left(\psi_{j, p_{n}}^{0}\right)^{\prime}$ are equicontinuous in $[0, R]$ and Arzelà-Ascoli Theorem implies (4.24). 
The locally uniform convergence established in Lemma 4.9 will be crucial to control the interactions among different scalings of the eigenfunction $\psi_{j, p}$. Adapting the proof of 6 , Lemma 3.7], we infer that

Lemma 4.10. If $\nu_{j}(p)<-\frac{1}{2}$, then for any $\delta>0$ there exist $K(\delta)>1$ and $p(\delta, K)>1$ such that

$$
\int_{G_{p}(K)} \frac{\left(\psi_{j, p}\right)^{2}}{r} d r \leq \delta, \quad \text { for } K \geq K(\delta) \text { and } p \geq p(\delta, K) .
$$

Here $G_{p}(K)$ is the set defined in (2.12).

Proof. By definition $G_{p}(K)=\bigcup_{i=0}^{m-1}\left[a_{i}, b_{i}\right]$ where we set

$$
\begin{aligned}
a_{i} & :=K \varepsilon_{i, p} \quad i=0, \ldots, m-1 \\
b_{i} & :=\left\{\begin{array}{lr}
\frac{1}{K} \varepsilon_{i+1, p} & i=0, \ldots, m-2 \\
1 & i=m-1
\end{array}\right.
\end{aligned}
$$

Thanks to Lemma 2.2 and the definition (4.10), one can chose $K(\delta)$ and $p_{1}(\delta)$ such that

$$
\max _{G_{p}(K)} f_{p} \leq \frac{\delta}{4 m}, \quad\left|K \eta^{i}(K)\left(\eta^{i}\right)^{\prime}(K)\right| \leq \frac{\delta}{16 m}, \quad\left|\frac{1}{K} \eta^{i}\left(\frac{1}{K}\right)\left(\eta^{i}\right)^{\prime}\left(\frac{1}{K}\right)\right| \leq \frac{\delta}{16 m}
$$

for every $K>K(\delta), p \geq p_{1}(\delta), i=0, \ldots m-1$.

Using $\psi_{j, p}$ as a test function in (3.7) and recalling the definition of $f_{p}$ in (2.11) we get

$$
\int_{a_{i}}^{b_{i}} \frac{\left(\psi_{j, p}\right)^{2}}{r} d r=-\frac{1}{\nu_{j}(p)} \int_{a_{i}}^{b_{i}}\left(r \psi_{j, p}^{\prime}\right)^{\prime} \psi_{j, p} d r-\frac{1}{\nu_{j}(p)} \int_{a_{i}}^{b_{i}} f_{p}(r) \frac{\left(\psi_{j, p}\right)^{2}}{r} d r .
$$

Let us estimate the two integrals in the right hand side of (4.30). Concerning the first one

$$
-\frac{1}{\nu_{j}(p)} \int_{a_{i}}^{b_{i}} f_{p}(r) \frac{\left(\psi_{j, p}\right)^{2}}{r} d r \leq 2 \max _{G_{p}(K)} f_{p} \int_{0}^{1} \frac{\left(\psi_{j, p}\right)^{2}}{r} d r \stackrel{\sqrt{3.11}}{=} 2 \max _{G_{p}(K)} f_{p} \leq \frac{\delta}{2 m}
$$

for every $K \geq K(\delta)$ and $p \geq p_{1}(\delta)$, thanks to (4.29).

Moreover integrating by parts

$$
\begin{aligned}
-\frac{1}{\nu_{j}(p)} \int_{a_{i}}^{b_{i}}\left(r \psi_{j, p}^{\prime}\right)^{\prime} \psi_{j, p} d r & =-\frac{1}{\nu_{j}(p)}\left[-\int_{a_{i}}^{b_{i}} r\left(\psi_{j, p}^{\prime}\right)^{2} d r+b_{i} \psi_{j, p}\left(b_{i}\right) \psi_{j, p}^{\prime}\left(b_{i}\right)-a_{i} \psi_{j, p}\left(a_{i}\right) \psi_{j, p}^{\prime}\left(a_{i}\right)\right] \\
& \leq 2\left|b_{i} \psi_{j, p}\left(b_{i}\right) \psi_{j, p}^{\prime}\left(b_{i}\right)\right|+2\left|a_{i} \psi_{j, p}\left(a_{i}\right) \psi_{j, p}^{\prime}\left(a_{i}\right)\right|
\end{aligned}
$$

since $\nu_{j}(p)<-\frac{1}{2}$. Observe that

$$
2 b_{m-1} \psi_{j, p}\left(b_{m-1}\right) \psi_{j, p}^{\prime}\left(b_{m-1}\right)=2 \psi_{j, p}(1) \psi_{j, p}^{\prime}(1)=0 .
$$

The other terms can be estimated by making use of Lemma 4.9. For $i=0, \ldots, m-2$, rescaling according to $\varepsilon_{i, p}$ gives

$$
2\left|b_{i} \psi_{j, p}\left(b_{i}\right) \psi_{j, p}^{\prime}\left(b_{i}\right)\right|=2 \frac{1}{K}\left|\psi_{j, p}^{i+1}\left(\frac{1}{K}\right)\left(\psi_{j, p}^{i+1}\right)^{\prime}\left(\frac{1}{K}\right)\right| \stackrel{\text { 4.23) }}{\leq} 2 \frac{1}{K}\left|\bar{\psi}_{j}^{i+1}\left(\frac{1}{K}\right)\left(\bar{\psi}_{j}^{i+1}\right)^{\prime}\left(\frac{1}{K}\right)\right|+\frac{\delta}{8 m}
$$

after chosing $p \geq p_{2}(\delta, K)$, for a suitable $p_{2}(\delta, K)$. Similarly for $i=0, \ldots, m-1$

$$
2\left|a_{i} \psi_{j, p}\left(a_{i}\right) \psi_{j, p}^{\prime}\left(a_{i}\right)\right|=2 K\left|\psi_{j, p}^{i}(K)\left(\psi_{j, p}^{i}\right)^{\prime}(K)\right| \leq 2 K\left|\bar{\psi}_{j}^{i}(K)\left(\bar{\psi}_{j}^{i}\right)^{\prime}(K)\right|+\frac{\delta}{8 m}
$$

for $p \geq p_{2}(\delta, K)$. Summing up, (4.32) becomes

$$
\begin{aligned}
& -\frac{1}{\nu_{j}(p)} \int_{a_{m-1}}^{b_{m-1}}\left(r \psi_{j, p}^{\prime}\right)^{\prime} \psi_{j, p} d r \leq 2 K\left|\bar{\psi}_{j}^{m-1}(K)\left(\bar{\psi}_{j}^{m-1}\right)^{\prime}(K)\right|+\frac{\delta}{8 m}, \\
& -\frac{1}{\nu_{j}(p)} \int_{a_{i}}^{b_{i}}\left(r \psi_{j, p}^{\prime}\right)^{\prime} \psi_{j, p} d r \leq 2 \frac{1}{K}\left|\bar{\psi}_{j}^{i+1}\left(\frac{1}{K}\right)\left(\bar{\psi}_{j}^{i+1}\right)^{\prime}\left(\frac{1}{K}\right)\right|+2 K\left|\bar{\psi}_{j}^{i}(K)\left(\bar{\psi}_{j}^{i}\right)^{\prime}(K)\right|+\frac{\delta}{4 m}
\end{aligned}
$$


if $i=0, \ldots m-2$. We remark that, according to Corollary 4.8 ( $(i i)$, at most one between the limit functions $\bar{\psi}_{j}^{i}$ and $\bar{\psi}_{j}^{i+1}$ differs from zero, and either $\bar{\psi}_{j}^{i}=A_{j} \eta^{i}$ or $\bar{\psi}_{j}^{i+1}=A_{j} \eta^{i+1}$, with $\left|A_{j}\right| \leq 1$. Therefore (4.29) implies that for every $i=0, \ldots m-1$

$$
-\frac{1}{\nu_{j}(p)} \int_{a_{i}}^{b_{i}}\left(r \psi_{j, p}^{\prime}\right)^{\prime} \psi_{j, p} d r \leq \frac{\delta}{2 m}
$$

for $K \geq K(\delta)$ and for every $p \geq p_{2}(\delta, K)$.

Substituting the estimates (4.31) and (4.33) into (4.30) we deduce that

$$
\int_{a_{i}}^{b_{i}} \frac{\left(\psi_{j, p}\right)^{2}}{r} d r \leq \frac{\delta}{m}
$$

for $K \geq K(\delta)$ and for every $p \geq \max \left\{p_{1}(\delta), p_{2}(\delta, K)\right\}$. The conclusion follows summing up for $i=0, \ldots, m-1$.

4.3. Proof of Proposition 4.3. Proposition 4.3 follows by adapting the arguments in 7 , Proposition 3.4], which concernes the case of two nodal zones. For the reader's comprehension we report a detailed proof. First we obtain an estimate from above of $\nu_{1}(p)$ in Lemma 4.11. Next we conclude the proof relying on the general convergence result in Proposition 4.7 and in particular on Corollary 4.8 and Lemma 4.9.

Lemma 4.11.

$$
\limsup _{p \rightarrow \infty} \nu_{1}(p) \leq \beta^{m-1}
$$

Proof. From the variational characterization (3.9), it suffices to exhibit for every $0<\varepsilon<1$ a sequence $\varphi_{p} \in \mathcal{H}_{0 \text {,rad }}$ such that

$$
\nu_{1}(p) \leq \frac{\int_{0}^{1} r\left(\left|\varphi_{p}^{\prime}\right|^{2}-V_{p} \varphi_{p}^{2}\right) d r}{\int_{0}^{1} r^{-1} \varphi_{p}^{2} d r} \leq \beta^{m-1}+\varepsilon
$$

if $p$ is large enough. So we pick a cut-off function $\Phi \in C_{0}^{\infty}(0, \infty)$ such that

$$
\begin{aligned}
& 0 \leq \Phi(r) \leq 1, \Phi(r)= \begin{cases}1 & \text { if } \frac{1}{R}<r<R, \\
0 & \text { if } 0 \leq r<\frac{1}{2 R} \text { or } r>2 R,\end{cases} \\
&\left|\Phi^{\prime}(r)\right| \leq \begin{cases}2 R & \text { if } \frac{1}{2 R}<r<\frac{1}{R}, \\
\frac{2}{R} & \text { if } R<r<2 R .\end{cases}
\end{aligned}
$$

Letting $\varepsilon_{p}=\varepsilon_{m-1, p}$ and $\eta=\eta^{m-1}$ as defined in (2.2) and (4.10), respectively, we set

$$
\varphi_{p}(r)=\eta\left(\frac{r}{\varepsilon_{p}}\right) \Phi\left(\frac{r}{\varepsilon_{p}}\right), \quad \text { as } r \in[0,1] .
$$

The function $\eta$ is increasing and decreasing on an interval $(0, a)$ and $(a, \infty)$ respectively, moreover $\lim _{s \rightarrow 0} \eta(s)=0, \lim _{s \rightarrow \infty} \eta(s)=0$, and $\int_{0}^{\infty} s^{-1} \eta^{2} d s=1$. So we can choose $R=R(\varepsilon)$ in such a way that

$$
\begin{array}{r}
\eta(s) \leq \eta\left(\frac{1}{R}\right)<\frac{\varepsilon}{4} \quad \text { for } s<\frac{1}{R} \quad \text { and } \quad \eta(s) \leq \eta(R)<\frac{\varepsilon}{4} \quad \text { for } s>R \\
\int_{0}^{\infty} s^{-1} \eta^{2} \Phi^{2} d s \geq \int_{\frac{1}{R}}^{R} s^{-1} \eta^{2} d s \geq 1-\varepsilon / 8 .
\end{array}
$$

Notice that since $\varepsilon_{p} \rightarrow 0$ we may assume w.l.g. that $p$ is so large that $1 / \varepsilon_{p}>2 R$, so that $\varphi_{p} \in \mathcal{H}_{0, \mathrm{rad}}$. 
Inserting the test function $\varphi_{p}$ in the variational characterization (3.9) of $\nu_{1}(p)$ we have

$$
\nu_{1}(p) \leq \frac{\int_{0}^{1} r\left(\left|\varphi_{p}^{\prime}\right|^{2}-V_{p} \varphi_{p}^{2}\right) d r}{\int_{0}^{1} r^{-1} \varphi_{p}^{2} d r}
$$

Next we estimate all the terms.

Using the relation $\left[(f g)^{\prime}\right]^{2}=f^{\prime}\left(f g^{2}\right)^{\prime}+f^{2}\left(g^{\prime}\right)^{2}$, scaling with respect to $\varepsilon$ and using the equation (4.8) satisfied by $\eta$ (recall that $\Phi$ has compact support) one gets

$$
\begin{aligned}
\int_{0}^{1} r\left|\varphi_{p}^{\prime}\right|^{2} d r & =\int_{0}^{1} r\left[\left(\eta\left(\frac{r}{\varepsilon_{p}}\right) \Phi\left(\frac{r}{\varepsilon_{p}}\right)\right)^{\prime}\right]^{2} d r \\
& =\frac{1}{\varepsilon_{p}} \int_{0}^{1} r \eta^{\prime}\left(\frac{r}{\varepsilon_{p}}\right)\left(\eta\left(\frac{r}{\varepsilon_{p}}\right) \Phi^{2}\left(\frac{r}{\varepsilon_{p}}\right)\right)^{\prime} d r+\frac{1}{\varepsilon_{p}^{2}} \int_{0}^{1} r \eta^{2}\left(\frac{r}{\varepsilon_{p}}\right)\left(\Phi^{\prime}\left(\frac{r}{\varepsilon_{p}}\right)\right)^{2} d r \\
& =\int_{0}^{\frac{1}{\varepsilon_{p}}} s \eta^{\prime}\left(\eta \Phi^{2}\right)^{\prime} d s+\int_{0}^{\frac{1}{\varepsilon_{p}}} s \eta^{2}\left(\Phi^{\prime}\right)^{2} d s \\
(4.40) & \stackrel{4.8}{=} \beta^{m-1} \int_{0}^{\infty} s^{-1} \eta^{2} \Phi^{2} d s+\int_{0}^{\infty} s e^{Z_{m-1}} \eta^{2} \Phi^{2} d s+\int_{0}^{\infty} s \eta^{2}\left(\Phi^{\prime}\right)^{2} d s
\end{aligned}
$$

and by the choice of $\Phi$ we have

$$
\begin{aligned}
\int_{0}^{\infty} s \eta^{2}\left(\Phi^{\prime}\right)^{2} d s & \leq 4 R^{2} \int_{\frac{1}{2 R}}^{\frac{1}{R}} s \eta^{2} d s+\frac{4}{R^{2}} \int_{R}^{2 R} s \eta^{2} d s \\
& \stackrel{\text { (4.377 }}{<} \frac{\varepsilon^{2} R^{2}}{4} \int_{\frac{1}{2 R}}^{\frac{1}{R}} s d s+\frac{\varepsilon^{2}}{4 R^{2}} \int_{R}^{2 R} s d s=\frac{3 \varepsilon^{2}}{4}<\frac{3 \varepsilon}{4} .
\end{aligned}
$$

Furthermore scaling with respect to $\varepsilon_{p}$, since $\frac{1}{\varepsilon_{p}}>2 R$ we get

$$
\int_{0}^{1} V_{p} \varphi_{p}^{2} d r=\int_{0}^{\infty} s V_{p}^{m-1} \eta^{2} \Phi^{2} d s
$$

and

$$
\int_{0}^{1} r^{-1} \varphi_{p}^{2} d r=\int_{0}^{\infty} s^{-1} \eta^{2} \Phi^{2} d s
$$

Inserting (4.40), (4.41), (4.42) and (4.43) in (4.39) we obtain

$$
\begin{aligned}
\nu_{1}(p) & <\beta^{m-1}+\frac{\int_{0}^{\infty} s\left(V_{p}^{m-1}-e^{Z_{m-1}}\right) \eta^{2} \Phi^{2} d s+\frac{3 \varepsilon}{4}}{\int_{0}^{\infty} s^{-1} \eta^{2} \Phi^{2} d s} \\
& \frac{4.38}{<} \beta^{m-1}+\frac{\int_{0}^{+\infty} s\left|e^{Z_{m-1}}-V_{p}^{m-1}\right| \eta^{2} \Phi^{2} d s+\frac{3}{4} \varepsilon}{1-\varepsilon / 8} .
\end{aligned}
$$

On the other hand by the properties of $\Phi$ we have

$$
\int_{0}^{+\infty} s\left|V_{p}^{m-1}-e^{Z_{m-1}}\right| \eta^{2} \Phi^{2} d s \leq \sup _{\left(\frac{1}{2 R}, 2 R\right)}\left|V_{p}^{m-1}-e^{Z_{m-1}}\right| \int_{\frac{1}{2 R}}^{2 R} s \eta^{2} d s
$$

and since $V_{p}^{m-1} \rightarrow e^{Z_{m-1}}$ uniformly on $\left[\frac{1}{2 R}, 2 R\right]$ we can take $p_{\varepsilon}$ in dependence by $\varepsilon$ and $R(\varepsilon)$ large enough such that

$$
\sup _{\left(\frac{1}{2 R}, 2 R\right)}\left|V_{p}^{m-1}-e^{Z_{m-1}}\right| \leq \frac{\varepsilon}{8 \int_{\frac{1}{2 R}}^{2 R} s \eta^{2} d s} \quad \text { for } p>p_{\varepsilon},
$$

which concludes the proof of (4.34). 
Proof of Proposition 4.3. By Lemma 4.11 and (4.12)

$$
\limsup _{p \rightarrow+\infty} \nu_{1}(p) \leq \beta^{m-1}<\beta^{i} \quad i=0, \ldots, m-2 .
$$

As a consequence, Corollary 4.8 ( $(i)$ implies that

$$
\bar{\psi}_{1}^{i} \equiv 0 \quad \text { as } i=0, \ldots m-2 .
$$

So, by Corollary 4.8 (ii), Proposition 4.3 is proved after checking that

$$
\bar{\psi}_{1}^{m-1} \not \equiv 0 \text {. }
$$

To this aim we fix $\delta>0$ such that $\delta<-\beta_{m-1} / 3$ and $K=K(\delta)$ and $p(\delta)$ as in Lemma 2.2 By the definition of $\nu_{1}(p)$ it follows that

$$
\begin{aligned}
-\nu_{1}(p) & =-\int_{0}^{1} r\left[\left(\psi_{1, p}^{\prime}\right)^{2}-V_{p}\left(\psi_{1, p}\right)^{2}\right] d r \\
& \leq \int_{0}^{1} r V_{p}\left(\psi_{1, p}\right)^{2} d r \\
& =\int_{G_{p}(K)} r V_{p}\left(\psi_{1, p}\right)^{2} d r+\int_{0}^{K \varepsilon_{0, p}} r V_{p}\left(\psi_{1, p}\right)^{2} d r+\sum_{i=1}^{m-1} \int_{\frac{1}{K} \varepsilon_{i, p}}^{K \varepsilon_{i, p}} r V_{p}\left(\psi_{1, p}\right)^{2} d r \\
& =I_{1}(p)+I_{2}(p)+I_{3}(p)
\end{aligned}
$$

The normalization of the eigenfunction and the estimate obtained in Lemma 2.2 assure that

$$
\begin{aligned}
I_{1}(p) & =\int_{G_{p}(K)} r V_{p}\left(\psi_{1, p}\right)^{2} d r=\int_{G_{p}(K)} f_{p} \frac{\left(\psi_{1, p}\right)^{2}}{r} d r \\
& \leq \sup _{G_{p}(K)} f_{p} \int_{0}^{1} \frac{\left(\psi_{1, p}\right)^{2}}{r} d r=\sup _{G_{p}(K)} f_{p} \leq \delta
\end{aligned}
$$

for $p \geq p(\delta)$.

Observe that, by Lemma 4.9, $\psi_{1, p}^{i} \rightarrow \bar{\psi}_{1}^{i}$ in $C_{\mathrm{loc}}^{1}(0,+\infty)$ for $i=1, \ldots m-1$, and in $C_{\text {loc }}^{1}[0,+\infty)$ for $i=0$. Indeed $\bar{\nu}_{1}:=\limsup _{p \rightarrow+\infty} \nu_{1}(p) \leq \beta^{m-1} \leq \beta^{1}<-25$ by (4.12). Furthermore, by (4.7) and (4.6),$V_{p}^{i} \rightarrow e^{Z_{i}}$ in $C_{\mathrm{loc}}^{0}(0,+\infty)$ for $i=1, \ldots, m-1$, or respectively in $C_{\mathrm{loc}}^{0}[0,+\infty)$ for $i=0$. Hence, rescaling the second integral according to $\varepsilon_{0, p}$ gives

$$
I_{2}(p)=\int_{0}^{K \varepsilon_{0, p}} r V_{p}\left(\psi_{1, p}\right)^{2} d r=\int_{0}^{K} r V_{p}^{0}\left(\psi_{1, p}^{0}\right)^{2} d r=\int_{0}^{K} r e^{Z_{0}}\left(\bar{\psi}_{1}^{0}\right)^{2} d r+o_{p}(1) \stackrel{\text { (4.44) }}{\leq} \delta,
$$

if $p \geq p_{2}(\delta)$. Similarly, for what concerns the third term,

$$
\begin{aligned}
I_{3}(p) & =\sum_{i=1}^{m-1} \int_{\frac{1}{K} \varepsilon_{i, p}}^{K \varepsilon_{i, p}} r V_{p}\left(\psi_{1, p}\right)^{2} d r=\sum_{i=1}^{m-1} \int_{\frac{1}{K}}^{K} r V_{p}^{i}\left(\psi_{1, p}^{i}\right)^{2} d r \\
& =\sum_{i=1}^{m-1} \int_{\frac{1}{K}}^{K} r e^{Z_{i}}\left(\bar{\psi}_{1}^{i}\right)^{2} d r+o_{p}(1) \\
& \stackrel{\text { 4.444 }}{\leq} \int_{\frac{1}{K}}^{K} r e^{Z_{m-1}}\left(\bar{\psi}_{1}^{m-1}\right)^{2} d r+\delta,
\end{aligned}
$$

for $p \geq p_{3}(\delta)$. Summing up, taking $\bar{p}=\max \left\{p(\delta), p_{2}(\delta), p_{3}(\delta)\right\}$ we have

$$
\int_{\frac{1}{K}}^{K} r e^{Z_{m-1}}\left(\bar{\psi}_{1}^{m-1}\right)^{2} d r \geq-\nu_{1}(p)-3 \delta \text { for } p>\bar{p}
$$


so, passing to the liminf and using Lemma 4.11

$$
\int_{\frac{1}{K}}^{K} r e^{Z_{m-1}}\left(\bar{\psi}_{1}^{m-1}\right)^{2} d r \geq-\limsup _{p \rightarrow+\infty} \nu_{1}(p)-3 \delta \geq-\beta^{m-1}-3 \delta>0
$$

by the choice of $\delta$. Hence $\bar{\psi}_{1}^{m-1} \neq 0$, concluding the proof.

4.4. Proof of Proposition 4.4. Computing the limits of the subsequent eigenvalues is more involved, and it is done in an iterative way. Similarly as in Section 4.3, also here we follow a two step scheme: first we obtain an estimate from above by producing a suitable test function (Lemma 4.12), then we conclude the proof of Proposition 4.4 by exploiting the convergence results in Proposition 4.7 and taking advantage of the orthogonality condition (3.11).

Lemma 4.12. Let $h \in\{2, \ldots, m\}$ and assume that Theorem 4.2 holds true for any $j=$ $1, \ldots, h-1$. Then

$$
\limsup _{p \rightarrow \infty} \nu_{h}(p) \leq \beta^{m-h}
$$

Proof. By the variational characterization (3.10), it suffices to exhibit for every $0<\varepsilon<1$ a function $\varphi_{p} \in \mathcal{H}_{0, \mathrm{rad}}, \varphi_{p} \perp \psi_{1, p}, \psi_{2, p}, \ldots, \psi_{h-1, p}$ such that

$$
\frac{\int_{0}^{1} r\left(\left|\varphi_{p}^{\prime}\right|^{2}-V_{p} \varphi_{p}^{2}\right) d r}{\int_{0}^{1} r^{-1} \varphi_{p}^{2} d r} \leq \beta^{m-h}+\varepsilon
$$

if $p$ is large enough. Let $\Phi=\Phi_{R}$ be the cut-off function defined in (4.35), $\varepsilon_{p}=\varepsilon_{m-h, p}$ and $\eta=\eta^{m-h}$ as defined in (2.2) and (4.10), respectively, and set

$$
\varphi_{p}(r)=\eta\left(\frac{r}{\varepsilon_{p}}\right) \Phi\left(\frac{r}{\varepsilon_{p}}\right)+\sum_{j=1}^{h-1} a_{j, p} \psi_{j, p}, \quad \text { as } r \in[0,1],
$$

with $R=R(\varepsilon)$ satisfying (4.37), (4.38) and $a_{j, p} \in \mathbb{R}$ choosen so that $\varphi_{p} \perp \psi_{1, p}, \psi_{2, p}, \ldots, \psi_{h-1, p}$, namely:

$$
a_{j, p}:=-\int_{0}^{1} r^{-1} \psi_{j, p}(r) \eta\left(\frac{r}{\varepsilon_{p}}\right) \Phi\left(\frac{r}{\varepsilon_{p}}\right) d r .
$$

Notice that since $\varepsilon_{p} \rightarrow 0$ we may assume w.l.g. that $p$ is so large that $1 / \varepsilon_{p}>2 R$, so that $\varphi_{p} \in \mathcal{H}_{0, \mathrm{rad}}$.

Furthermore

$$
a_{j, p} \rightarrow 0 \text { as } p \rightarrow \infty
$$

Indeed, rescaling w.r.t. $\varepsilon_{p}$, using that $\Phi$ has compact support and that the interval $\left(\frac{r_{m-h, p}}{\varepsilon_{p}}, \frac{r_{m-h+1, p}}{\varepsilon_{p}}\right)$ invades $(0, \infty)$ by (2.8), we can write for $p$ large

$$
\begin{aligned}
\int_{0}^{1} r^{-1} \psi_{j, p}(r) \eta\left(\frac{r}{\varepsilon_{p}}\right) \Phi\left(\frac{r}{\varepsilon_{p}}\right) d r= & \int_{0}^{r_{m-h, p}} r^{-1} \psi_{j, p}(r) \eta\left(\frac{r}{\varepsilon_{p}}\right) \Phi\left(\frac{r}{\varepsilon_{p}}\right) d r \\
& +\int_{r_{m-h, p}}^{r_{m-h+1, p}} r^{-1} \psi_{j, p}(r) \eta\left(\frac{r}{\varepsilon_{p}}\right) \Phi\left(\frac{r}{\varepsilon_{p}}\right) d r \\
& +\int_{r_{m-h+1, p}}^{1} r^{-1} \psi_{j, p}(r) \eta\left(\frac{r}{\varepsilon_{p}}\right) \Phi\left(\frac{r}{\varepsilon_{p}}\right) d r \\
= & \int_{r_{m-h, p} / \varepsilon_{p}}^{r_{m-h+1, p} / \varepsilon_{p}} s^{-1} \psi_{j, p}^{m-h} \eta \Phi d s
\end{aligned}
$$


(4.50) then follows passing to the limit as $p \rightarrow \infty$ and using that $\psi_{j, p}^{m-h} \rightarrow \bar{\psi}_{j}^{m-h}$ weakly in $\mathcal{D}_{\text {rad }}$ by Proposition 4.7 and that $\bar{\psi}_{j}^{m-h}=0$ for $j=1, \ldots, h-1$ by assumption.

We want to estimate all the integrals in the left hand side of (4.47). Observe that

$$
\begin{aligned}
\int_{0}^{1} r\left|\varphi_{p}^{\prime}\right|^{2} d r= & \int_{0}^{1} r\left[\left(\eta\left(\frac{r}{\varepsilon_{p}}\right) \Phi\left(\frac{r}{\varepsilon_{p}}\right)\right)^{\prime}\right]^{2} d r+\sum_{j=1}^{h-1} a_{j, p}^{2} \int_{0}^{1} r\left(\psi_{j, p}^{\prime}\right)^{2} d r \\
& +2 \sum_{j=1}^{h-1} a_{j, p} \int_{0}^{1} r\left(\eta\left(\frac{r}{\varepsilon_{p}}\right) \Phi\left(\frac{r}{\varepsilon_{p}}\right)\right)^{\prime} \psi_{j, p}^{\prime} d r \\
& +\sum_{j, \ell=1, j \neq \ell}^{h-1} a_{j, p} a_{\ell, p} \int_{0}^{1} r \psi_{j, p}^{\prime} \psi_{\ell, p}^{\prime} d r \\
= & A_{p}+B_{p}+C_{p}+D_{p}
\end{aligned}
$$

and similarly that

$$
\begin{aligned}
\int_{0}^{1} r V_{p} \varphi_{p}^{2} d r= & \int_{0}^{1} r V_{p}(r) \eta\left(\frac{r}{\varepsilon_{p}}\right)^{2} \Phi\left(\frac{r}{\varepsilon_{p}}\right)^{2} d r+\sum_{j=1}^{h-1} a_{j, p}^{2} \int_{0}^{1} r V_{p}(r)\left(\psi_{j, p}\right)^{2} d r \\
& +2 \sum_{j=1}^{h-1} a_{j, p} \int_{0}^{1} r V_{p}(r) \eta\left(\frac{r}{\varepsilon_{p}}\right) \Phi\left(\frac{r}{\varepsilon_{p}}\right) \psi_{j, p} d r \\
& +\sum_{j, \ell=1, j \neq \ell}^{h-1} a_{j, p} a_{\ell, p} \int_{0}^{1} r V_{p}(r) \psi_{j, p} \psi_{\ell, p} d r \\
= & E_{p}+F_{p}+G_{p}+H_{p} .
\end{aligned}
$$

The same computations as in Lemma 4.11 (see (4.40) and (4.42) show that

$$
\begin{aligned}
A_{p}-E_{p}= & \beta^{m-h} \int_{0}^{\infty} s^{-1} \eta^{2} \Phi^{2} d s+\int_{0}^{\infty} s e^{Z_{m-h}} \eta^{2} \Phi^{2} d s+\int_{0}^{\infty} s \eta^{2}\left(\Phi^{\prime}\right)^{2} d s \\
& -\int_{0}^{\infty} s V_{p}^{m-h} \eta^{2} \Phi^{2} d s .
\end{aligned}
$$

Next using that $\psi_{j, p}$ solves (3.7) and (3.11), and recalling the definition of $a_{j, p}$ in (4.49), we have

$$
\begin{aligned}
B_{p}-F_{p} & =\sum_{j=1}^{h-1} a_{j, p}^{2} \nu_{j}(p) ; \\
C_{p}-G_{p} & =2 \sum_{j=1}^{h-1} a_{j, p} \nu_{j}(p) \int_{0}^{1} r^{-1} \eta\left(\frac{r}{\varepsilon_{p}}\right) \Phi\left(\frac{r}{\varepsilon_{p}}\right) \psi_{j, p} d r \\
\stackrel{(4.49)}{=} & -2 \sum_{j=1}^{h-1} a_{j, p}^{2} \nu_{j}(p) ; \\
D_{p}-H_{p} & =\sum_{j, \ell=1, j \neq \ell}^{h-1} a_{j, p} a_{\ell, p} \nu_{\ell}(p) \int_{0}^{1} r^{-1} \psi_{j, p} \psi_{\ell, p} d r=0 .
\end{aligned}
$$


Hence substituting (4.54), (4.55), (4.56) and (4.57) in (4.51) and (4.52) we infer:

$$
\begin{aligned}
\int_{0}^{1} r\left|\varphi_{p}^{\prime}\right|^{2} d r-\int_{0}^{1} r V_{p} \varphi_{p}^{2} d r= & \beta^{m-h} \int_{0}^{\infty} s^{-1} \eta^{2} \Phi^{2} d s+\int_{0}^{\infty} s \eta^{2}\left(\Phi^{\prime}\right)^{2} d s \\
& +\int_{0}^{\infty} s\left(e^{Z_{m-h}}-V_{p}^{m-h}\right) \eta^{2} \Phi^{2} d s \\
& -\sum_{j=1}^{h-1} a_{j, p}^{2} \nu_{j}(p) .
\end{aligned}
$$

On the other hand using once more (3.11) and (4.49), rescaling with respect to $\varepsilon_{p}$ and using the properties of $\Phi$ it also follows that

$$
\begin{aligned}
\int_{0}^{1} r^{-1} \varphi_{p}^{2} d r= & \int_{0}^{1} r^{-1}\left(\eta\left(\frac{r}{\varepsilon_{p}}\right) \Phi\left(\frac{r}{\varepsilon_{p}}\right)\right)^{2} d r+\sum_{j, \ell=1}^{h-1} a_{j, p} a_{\ell, p} \int_{0}^{1} r^{-1} \psi_{j, p} \psi_{\ell, p} d r \\
& +2 \sum_{j=1}^{h-1} a_{j, p} \int_{0}^{1} r^{-1} \psi_{j, p} \eta\left(\frac{r}{\varepsilon_{p}}\right) \Phi\left(\frac{r}{\varepsilon_{p}}\right) d r \\
= & \int_{0}^{1} r^{-1}\left(\eta\left(\frac{r}{\varepsilon_{p}}\right) \Phi\left(\frac{r}{\varepsilon_{p}}\right)\right)^{2} d r-\sum_{j=1}^{h-1} a_{j, p}^{2} \\
= & \int_{0}^{\infty} s^{-1} \eta^{2} \Phi^{2} d s-\sum_{j=1}^{h-1} a_{j, p}^{2} .
\end{aligned}
$$

Inserting (4.58) and (4.59) into the 1.h.s. of (4.47) we get

$$
\begin{aligned}
& \frac{\int_{0}^{1} r\left(\left|\varphi_{p}^{\prime}\right|^{2}-V_{p} \varphi_{p}^{2} d r\right) d r}{\int_{0}^{1} r^{-1} \varphi_{p}^{2} d r}= \\
& =\beta^{m-h}+\frac{\int_{0}^{\infty} s\left(V_{p}^{m-h}-e^{Z_{m-h}}\right) \eta^{2} \Phi^{2} d s+\int_{0}^{\infty} s \eta^{2}\left(\Phi^{\prime}\right)^{2} d s-\sum_{j=1}^{h-1} a_{j, p}^{2}\left(\nu_{j}(p)-\beta^{m-h}\right)}{\int_{0}^{\infty} s^{-1} \eta^{2} \Phi^{2} d s-\sum_{j=1}^{h-1} a_{j, p}^{2}} \\
& \stackrel{+4.50)}{=} \beta^{m-h}+\frac{\int_{0}^{\infty} s\left(V_{p}^{m-h}-e^{Z_{m-h}}\right) \eta^{2} \Phi^{2} d s+\int_{0}^{\infty} s \eta^{2}\left(\Phi^{\prime}\right)^{2} d s+o(1)}{\int_{0}^{\infty} s^{-1} \eta^{2} \Phi^{2} d s+o(1)} \\
& \leq \underset{14.38)}{\leq} \beta^{m-h}+\frac{\int_{0}^{\infty} s\left(V_{p}^{m-h}-e^{Z_{m-h}}\right) \eta^{2} \Phi^{2} d s+\frac{3}{4} \varepsilon+o(1)}{1-\frac{\varepsilon}{8}+o(1)} .
\end{aligned}
$$

On the other hand, similarly as at the end of the proof of Lemma 4.11, one can prove that

$$
\int_{0}^{+\infty} s\left|V_{p}^{m-h}-e^{Z_{m-h}}\right| \eta^{2} \Phi^{2} d s \leq \sup _{\left(\frac{1}{2 R}, 2 R\right)}\left|V_{p}^{m-h}-e^{Z_{m-h}}\right| \int_{\frac{1}{2 R}}^{2 R} s \eta^{2} d s \leq \frac{\varepsilon}{8}
$$

which concludes the proof of (4.47).

Next we conclude the proof of Proposition 4.4 by exploiting the orthogonality condition (3.11), which allows to pick up, among all the rescaled functions introduced in (4.1), the only one which has a nontrivial limit.

Proof of Proposition 4.4. Fix $h \in\{2, \ldots, m-1\}$, we want to prove that:

$$
\lim _{p \rightarrow+\infty} \nu_{h}(p)=\beta^{m-h}
$$


and that there exists $A_{h} \neq 0$ such that

$$
\begin{aligned}
& \bar{\psi}_{h}^{m-h}=A_{h} \eta^{m-h} ; \\
& \bar{\psi}_{h}^{i}=0, \quad i=0, \ldots, m-1, i \neq m-h .
\end{aligned}
$$

By Lemma 4.12 and (4.12) $\limsup _{p \rightarrow+\infty} \nu_{h}(p) \leq \beta^{m-h}<\beta^{m-i}$ for $i=h+1, \ldots m$, then Corollary 4.8 ( $(i)$ implies that

$$
\bar{\psi}_{h}^{m-i}=0, \quad i=h+1, \ldots m
$$

Furthermore the claim follows by showing that $\bar{\psi}_{h}^{m-h} \neq 0$, thanks to Corollary 4.8 (ii). So we assume by contradiction that

$$
\bar{\psi}_{h}^{m-h}=0
$$

As a preliminary step, we will deduce from (4.61), (4.62) that there exists $\kappa \in\{1, \ldots h-1\}$ such that

$$
\bar{\psi}_{h}^{m-\kappa} \neq 0
$$

In order to prove (4.63) let us fix $\delta>0$ such that $\delta<-\beta^{m-h} / 3$ and $K=K(\delta)$ as in Lemma 2.2. By the definition of of $\nu_{h}(p)$ it follows that

$$
\begin{aligned}
-\nu_{h}(p) & =-\int_{0}^{1} r\left[\left(\psi_{h, p}^{\prime}\right)^{2}-V_{p}\left(\psi_{h, p}\right)^{2}\right] d r \\
& \leq \int_{0}^{1} r V_{p}\left(\psi_{h, p}\right)^{2} d r \\
& =\int_{G_{p}(K)} r V_{p}\left(\psi_{h, p}\right)^{2} d r+\int_{0}^{K \varepsilon_{0, p}} r V_{p}\left(\psi_{h, p}\right)^{2} d r+\sum_{i=1}^{m-1} \int_{\frac{1}{K} \varepsilon_{i, p}}^{K \varepsilon_{i, p}} r V_{p}\left(\psi_{h, p}\right)^{2} d r \\
& =I_{1}(p)+I_{2}(p)+I_{3}(p) .
\end{aligned}
$$

We estimate these three terms with arguments similar to the ones exploited in the proof of Proposition 4.3. Indeed the normalization of the eigenfunction and the estimate obtained in Lemma 2.2 assure that

$$
\begin{aligned}
I_{1}(p) & =\int_{G_{p}(K)} r V_{p}\left(\psi_{h, p}\right)^{2} d r=\int_{G_{p}(K)} f_{p} \frac{\left(\psi_{h, p}\right)^{2}}{r} d r \\
& \leq \sup _{G_{p}(K)} f_{p} \int_{0}^{1} \frac{\left(\psi_{h, p}\right)^{2}}{r} d r=\sup _{G_{p}(K)} f_{p} \leq \delta
\end{aligned}
$$

for $p \geq p_{1}(\delta)$.

Moreover, by Lemma 4.9.

$$
\begin{aligned}
\psi_{h, p}^{i} \rightarrow \bar{\psi}_{h}^{i} \quad & \text { in } C_{\mathrm{loc}}^{1}(0,+\infty) \text { for } i=1, \ldots m-1, \\
& \text { in } C_{\mathrm{loc}}^{1}[0,+\infty) \text { for } i=0 .
\end{aligned}
$$

Indeed $\bar{\nu}_{h}:=\lim \sup _{p \rightarrow+\infty} \nu_{h}(p) \leq \beta^{m-h} \leq \beta^{1}<-25$ by (4.12).

Furthermore, by (4.7) and (4.6),$V_{p}^{i} \rightarrow e^{Z_{i}}$ in $C_{\mathrm{loc}}^{0}(0,+\infty)$ for $i=1, \ldots, m-1$, in $C_{\mathrm{loc}}^{0}[0,+\infty)$ for $i=0$.

Hence, rescaling the second integral according to $\varepsilon_{0, p}$ gives

$$
I_{2}(p)=\int_{0}^{K \varepsilon_{0, p}} r V_{p}\left(\psi_{h, p}\right)^{2} d r=\int_{0}^{K} r V_{p}^{0}\left(\psi_{h, p}^{0}\right)^{2} d r \stackrel{\text { (4.24) }}{=} \int_{0}^{K} r e^{Z_{0}}\left(\bar{\psi}_{h}^{0}\right)^{2} d r+o_{p}(1) \stackrel{\sqrt[4.61]{\leq}}{\leq} \delta,
$$


if $p \geq p_{2}(\delta, K)$. Similarly, for what concerns the third term,

$$
\begin{array}{cc}
I_{3}(p)= & \sum_{i=1}^{m-1} \int_{\frac{1}{K} \varepsilon_{i, p}}^{K \varepsilon_{i, p}} r V_{p}\left(\psi_{h, p}\right)^{2} d r=\sum_{i=1}^{m-1} \int_{\frac{1}{K}}^{K} r V_{p}^{i}\left(\psi_{h, p}^{i}\right)^{2} d r \\
\stackrel{4.20}{=} & \sum_{i=1}^{m-1} \int_{\frac{1}{K}}^{K} r e^{Z_{i}}\left(\bar{\psi}_{h}^{i}\right)^{2} d r+o_{p}(1) \\
\stackrel{4.61, \text { (4.62) }}{\leq} & \sum_{\kappa=1}^{h-1} \int_{\frac{1}{K}}^{K} r e^{Z_{m-\kappa}}\left(\bar{\psi}_{h}^{m-\kappa}\right)^{2} d r+\delta,
\end{array}
$$

for $p \geq p_{3}(\delta, K)$. Summing up we then get

$$
\sum_{\kappa=1}^{h-1} \int_{\frac{1}{K}}^{K} r e^{Z_{m-\kappa}}\left(\bar{\psi}_{h}^{m-\kappa}\right)^{2} d r \geq-\nu_{h}(p)-3 \delta, \quad \text { for } p \geq \bar{p}:=\max \left\{p_{1}(\delta), p_{2}(\delta, K), p_{3}(\delta, K)\right\} \text {. }
$$

Passing to the liminf as $p \rightarrow \infty$ and using Lemma 4.12 we get

$$
\sum_{\kappa=1}^{h-1} \int_{\frac{1}{K}}^{K} r e^{Z_{m-\kappa}}\left(\bar{\psi}_{h}^{m-\kappa}\right)^{2} d r \geq-\limsup _{p \rightarrow \infty} \nu_{h}(p)-3 \delta \geq-\beta_{m-h}-3 \delta>0,
$$

by the choice of $\delta$, which gives (4.63).

By (4.63), Corollary 4.8 (ii) implies that there exists $A_{h} \neq 0$ such that

$$
\begin{aligned}
& \bar{\psi}_{h}^{m-\kappa}=A_{h} \eta^{m-\kappa} \\
& \bar{\psi}_{h}^{i}=0, \quad i=0, \ldots, m-1, i \neq m-\kappa .
\end{aligned}
$$

Furthermore, since by assumption Theorem 4.2 holds true for any index below $h$, there exists $A_{\kappa} \neq 0$ such that

$$
\begin{aligned}
& \bar{\psi}_{\kappa}^{m-\kappa}=A_{\kappa} \eta^{m-\kappa} \\
& \bar{\psi}_{\kappa}^{i}=0, \quad i=0, \ldots, m-1, i \neq m-\kappa .
\end{aligned}
$$

We conclude the proof by showing that (4.66) and (4.68) can not hold at the same time, due to the orthogonality condition (3.11).

Observe also that by Lemma 4.9 .

$$
\begin{aligned}
\psi_{\kappa, p}^{i} \rightarrow \bar{\psi}_{\kappa}^{i} \quad & \text { in } C_{\mathrm{loc}}^{1}(0,+\infty) \text { for } i=1, \ldots m-1, \\
& \text { in } C_{\mathrm{loc}}^{1}[0,+\infty) \text { for } i=0,
\end{aligned}
$$

since by assumption $\bar{\nu}_{\kappa}:=\lim _{p \rightarrow+\infty} \nu_{\kappa}(p)=\beta^{m-\kappa}$ and by (4.12) $\beta^{m-\kappa} \leq-25$.

Then since $\psi_{\kappa, p} \perp \psi_{h, p}$, for any $K>1$ and for any $p>1$ we write

$$
\begin{aligned}
& 0=\int_{0}^{1} \frac{\psi_{\kappa, p} \psi_{h, p}}{r} d r \\
& =\int_{G_{p}(K)} \frac{\psi_{\kappa, p} \psi_{h, p}}{r} d r+\int_{0}^{K \varepsilon_{0, p}} \frac{\psi_{\kappa, p} \psi_{h, p}}{r} d r+\sum_{\substack{i=1 \\
i \neq m-\kappa}}^{m-1} \int_{\frac{1}{K} \varepsilon_{i, p}}^{K \varepsilon_{i, p}} \frac{\psi_{\kappa, p} \psi_{h, p}}{r} d r+\int_{\frac{1}{K} \varepsilon_{m-\kappa, p}}^{K \varepsilon_{m-\kappa, p}} \frac{\psi_{\kappa, p} \psi_{h, p}}{r} d r \\
& =I(4 p, \mathcal{X}))+I_{2}(p, K)+I_{3}(p, K)+I_{4}(p, K) .
\end{aligned}
$$

First, as both (4.66) and (4.68) hold true, we can take $\delta>0$ so that

$$
\delta<\min \left\{\frac{1}{4}, \frac{\left|A_{\kappa} A_{h}\right|}{4}\right\} .
$$


Since $\int_{0}^{\infty} \frac{\left(\eta^{m-\kappa}\right)^{2}}{r} d r=1$, there exists $K_{1}(\delta)>1$ such that

$$
\int_{\frac{1}{K}}^{K} \frac{\left(\eta^{m-\kappa}\right)^{2}}{r} d r \geq 1-\delta, \quad \forall K \geq K_{1}(\delta) .
$$

Moreover, by Hölder inequality and Lemma 4.10, we can take $K>K_{1}(\delta)$ and accordingly $p_{1}(\delta, K)$ such that

$$
\left|I_{1}(p, K)\right|:=\left|\int_{G_{p}(K)} \frac{\psi_{\kappa, p} \psi_{h, p}}{r} d r\right| \leq\left[\int_{G_{p}(K)} \frac{\left(\psi_{\kappa, p}\right)^{2}}{r} d r\right]^{\frac{1}{2}}\left[\int_{G_{p}(K)} \frac{\left(\psi_{h, p}\right)^{2}}{r} d r\right]^{\frac{1}{2}} \leq \delta
$$

for every $p>p_{1}(\delta, K)$.

For the second term we rescale according to the parameter $\varepsilon_{0, p}$ and exploits the $C_{\text {loc }}^{1}[0, \infty)$ convergences of $\psi_{h, p}^{0}$ to $\bar{\psi}_{h}^{0}$ in (4.65) and of $\psi_{\kappa, p}^{0}$ to $\bar{\psi}_{\kappa}^{0}$ in (4.71), we then get

$$
\begin{aligned}
\left|I_{2}(p, K)\right| & :=\left|\int_{0}^{K \varepsilon_{0, p}} \frac{\psi_{\kappa, p} \psi_{h, p}}{r} d r\right|=\left|\int_{0}^{K} \frac{\psi_{\kappa, p}^{0} \psi_{h, p}^{0}}{r} d r\right| \\
& =\left|\int_{0}^{K} \frac{\bar{\psi}_{\kappa}^{0} \bar{\psi}_{h}^{0}}{r} d r\right|+o_{p}(1)=o_{p}(1) \leq \delta,
\end{aligned}
$$

for any $p \geq p_{2}(\delta, K)$, where the last equality follows from the fact that $\bar{\psi}_{\kappa}^{0}=0$ by (4.69). Similarly (scaling with parameter $\varepsilon_{i, p}$ and exploiting the convergences in (4.64) and (4.70) we also get

$$
\begin{aligned}
\left|I_{3}(p, K)\right| & :=\left|\sum_{\substack{i=1 \\
i \neq m-\kappa}}^{m-1} \int_{\frac{1}{K} \varepsilon_{i, p}}^{K \varepsilon_{i, p}} \frac{\psi_{\kappa, p} \psi_{h, p}}{r} d r\right|=\left|\sum_{\substack{i=1 \\
i \neq m-\kappa}}^{m-1} \int_{\frac{1}{K}}^{K} \frac{\psi_{\kappa, p}^{i} \psi_{h, p}^{i}}{r} d r\right| \\
& \leq \sum_{\substack{i=1 \\
i \neq m-\kappa}}^{m-1}\left|\int_{\frac{1}{K}}^{K} \frac{\bar{\psi}_{\kappa}^{i} \bar{\psi}_{h}^{i}}{r} d r\right|+o_{p}(1)=o_{p}(1) \leq \delta,
\end{aligned}
$$

for any $p \geq p_{3}(\delta, K)$, where the last equality follows from the fact that $\bar{\psi}_{\kappa}^{i}=0$, for any $i=1, \ldots, m-1, i \neq m-\kappa$ by (4.69). Hence, substituting (4.75), (4.76), (4.77) into (4.72), one gets

$$
\left|I_{4}(p, K)\right| \leq 3 \delta, \quad \forall p \geq \max \left\{p_{1}(\delta), p_{2}(\delta, K), p_{3}(\delta, K)\right\} .
$$

On the other side, scaling with parameter $\varepsilon_{m-\kappa, p}$, passing to the limit thanks to (4.64) and (4.70) with $i=m-\kappa$, we also get

$$
\begin{aligned}
I_{4}(p, K) & :=\int_{\frac{1}{K} \varepsilon_{m-\kappa, p}}^{K \varepsilon_{m-\kappa, p}} \frac{\psi_{\kappa, p} \psi_{h, p}}{r} d r=\int_{\frac{1}{K}}^{K} \frac{\psi_{\kappa, p}^{m-\kappa} \psi_{h, p}^{m-\kappa}}{r} d r=\int_{\frac{1}{K}}^{K} \frac{\bar{\psi}_{\kappa}^{m-\kappa} \bar{\psi}_{h}^{m-\kappa}}{r} d r+o_{p}(1) \\
& =A_{\kappa} A_{h} \int_{\frac{1}{K}}^{K} \frac{\left(\eta^{m-\kappa}\right)^{2}}{r} d r+o_{p}(1),
\end{aligned}
$$

as $p \rightarrow+\infty$, where the last equality follows from (4.68) and (4.66). Eventually, passing to the limit for $p \rightarrow \infty$ yields

or equivalenty

$$
\left|A_{\kappa} A_{h}\right| \int_{\frac{1}{K}}^{K} \frac{\left(\eta^{m-\kappa}\right)^{2}}{r} d r \leq 3 \delta
$$

$$
\left|A_{\kappa} A_{h}\right| \leq \frac{3 \delta}{\int_{\frac{1}{K}}^{K} \frac{\left(\eta^{m-\kappa}\right)^{2}}{r} d r} \underset{14.74}{\leq} \frac{3 \delta}{1-\delta} .
$$

But this last inequality clashes with (4.73) because

$$
\frac{3 \delta}{1-\delta} \underset{\delta<\frac{\left|A_{\kappa} A_{h}\right|}{4}}{<} \frac{3}{4(1-\delta)}\left|A_{\kappa} A_{h}\right| \underset{\delta<\frac{1}{4}}{<}\left|A_{\kappa} A_{h}\right| .
$$


In that way we have reached a contradiction and the proof is completed.

4.5. Last eigenvalue: the proof of Proposition 4.5. Here we prove Proposition 4.5, thus ending the proof of Theorem 4.2 .

Proof of Proposition 4.5. Comparing the estimates (3.12) and (4.46) (for $h=m$ ) and recalling that $\beta_{0}=-1$ by (4.12) yields

$$
\lim _{p \rightarrow+\infty} \nu_{m}(p)=\beta^{0}=-1 .
$$

Proposition 4.7 and Corollary 4.8. (ii) give that

$$
\begin{array}{ll}
\psi_{m, p}^{0} \rightarrow A_{m} \eta^{0} & \\
\psi_{m, p}^{i} \rightarrow \bar{\psi}_{m}^{i}=0 & \text { for } i=1, \ldots m-1,
\end{array}
$$

where the convergence is weak in $\mathcal{D}_{\text {rad }}$, strong in $L_{\text {loc }}^{2}(0, \infty)$, and also strong in $C_{\text {loc }}^{1}(0, \infty)$ thanks to Lemma 4.9. It remains to check that the constant $A_{m}$ in (4.79) is not zero.

Let $\delta>0, K=K(\delta)$ and $p \geq p(\delta)$ where $K(\delta)$ and $p(\delta)$ are as in Lemma 2.2. Following the ideas in [7, Proposition 3.5], from the equation (3.7) we deduce that

$$
\begin{aligned}
-\nu_{m}(p) & =-\int_{0}^{1} r\left[\left(\psi_{m, p}^{\prime}\right)^{2}-V_{p}\left(\psi_{m, p}\right)^{2}\right] d r \leq \int_{0}^{1} r V_{p}\left(\psi_{m, p}\right)^{2} d r \\
& =\int_{G_{p}(K)} r V_{p}\left(\psi_{m, p}\right)^{2} d r+\int_{0}^{K \varepsilon_{0, p}} r V_{p}\left(\psi_{m, p}\right)^{2} d r+\sum_{i=1}^{m-1} \int_{\frac{1}{K} \varepsilon_{i, p}}^{K \varepsilon_{i, p}} r V_{p}\left(\psi_{m, p}\right)^{2} d r \\
& =I_{1}(p)+I_{2}(p)+I_{3}(p) .
\end{aligned}
$$

Hence the normalization (3.11) of the eigenfunction and the estimate obtained in Lemma 2.2 imply

$$
I_{1}(p)=\int_{G_{p}(K)} f_{p} \frac{\left(\psi_{m, p}\right)^{2}}{r} d r \leq \sup _{G_{p}(K)} f_{p} \leq \delta .
$$

Furthermore rescaling each integral according to $\varepsilon_{i, p}$ gives

$$
I_{3}(p)=\sum_{i=1}^{m-1} \int_{\frac{1}{K} \varepsilon_{i, p}}^{K \varepsilon_{i, p}} r V_{p}\left(\psi_{m, p}\right)^{2} d r=\sum_{i=1}^{m-1} \int_{\frac{1}{K}}^{K} r V_{p}^{i}\left(\psi_{m, p}^{i}\right)^{2} d r \leq \delta,
$$

for $p \geq p_{3}(\delta, K)$, thanks to (4.7) and (4.80).

Finally rescaling according to $\varepsilon_{0, p}$ and using the uniform convergence in (4.6) and the $L^{2}$ convergence in (4.79) one has

$$
\begin{aligned}
I_{2}(p) & =\int_{0}^{K} r V_{p}^{0}\left(\psi_{m, p}^{0}\right)^{2} d r \\
& =\left(A_{m}\right)^{2} \int_{0}^{K} r e^{Z_{0}}\left(\eta^{0}\right)^{2} d r+o_{p}(1) \leq\left(A_{m}\right)^{2} \int_{0}^{K} r e^{Z_{0}}\left(\eta^{0}\right)^{2} d r+\delta
\end{aligned}
$$

provided that $p \geq p_{2}(\delta, K)$. Notice that Lemma 4.9 does not guarantee the convergence in $C_{\text {loc }}^{1}[0, \infty)$, since $\beta_{0}=-1$.

Summing up we have showed that

$$
-\nu_{m}(p) \leq\left(A_{m}\right)^{2} \int_{0}^{K} r e^{Z_{0}}\left(\eta^{0}\right)^{2} d r+3 \delta,
$$

provided that $p \geq \max \left\{p(\delta), p_{2}(\delta, K), p_{3}(\delta, K)\right\}$. Eventually

$$
1=\limsup _{p \rightarrow \infty}\left(-\nu_{m}(p)\right) \leq\left(A_{m}\right)^{2} \int_{0}^{K} r e^{Z_{0}}\left(\eta^{0}\right)^{2} d r
$$


from which $A_{m} \neq 0$ follows.

\section{The Proof of TheOrem 1.1 IN The CASE $\alpha=0$}

In this section we compute the exact value of the Morse index of the radial solution $u_{p}$ of the Lane-Emden problem (2.1), proving that formula (1.14) holds if $p$ is sufficiently large.

This result follows directly from formula (3.13) and from the asymptotic behavior of the singular eigenvalues $\nu_{j}(p), j=1, \ldots, m-1$, as $p \rightarrow+\infty$, which has been stated in Theorem 1.2 (cfr. the more general version Theorem 4.2).

Proof of (1.14). Let $u_{p}$ be the solution to the Lane-Emden problem (2.1) having $m-1$ interior zeros. From formula (3.13) we know that the Morse index $\mathrm{m}\left(u_{p}\right)$ is given implicitly in terms of the negative radial eigenvalues $\nu_{j}(p), j=1, \ldots, m-1$, of the singular problem (3.7). Moreover from Theorem 4.2 we know that

$$
\sqrt{-\nu_{j}(p)} \rightarrow \frac{\theta_{m-j}}{2} \text { as } p \rightarrow+\infty, \text { for } j=1, \ldots m-1 .
$$

hence, recalling (2.4) we see that

$$
\left\lceil\sqrt{-\nu_{j}(p)}-1\right\rceil=\left[\frac{\theta_{m-j}}{2}\right]=4(m-j)+1
$$

for $p$ large. The conclusion follows from formula (3.13) and (5.1), indeed for $p$ large

$$
\mathrm{m}\left(u_{p}\right)=m+2 \sum_{i=1}^{m-1}\left[\frac{\theta_{i}}{2}\right]
$$

\section{The Proof of Theorem [1.1 In The CASE $\alpha>0$}

At last we exploit the connection between Lane-Emden and Hénon problem and show how the already performed analysis allows to compute also the Morse index of radial solutions to the Hénon problem, concluding the proof of Theorem 1.1. For every $\alpha>0$, we denote by $u_{\alpha, p}$ the unique radial solution to

$$
\begin{cases}-\Delta u=|x|^{\alpha}|u|^{p-1} u & \text { in } B \\ u=0 & \text { on } \partial B\end{cases}
$$

with $m$ nodal zones which is positive at the origin. In dimension $N=2$ radial solutions to (6.1) and (2.1) are linked via the transformation

$$
u_{p}(t)=\left(\frac{2}{2+\alpha}\right)^{\frac{2}{p-1}} u_{\alpha, p}(r), \quad t=r^{\frac{2+\alpha}{2}},
$$

where, as in the previous sections, $u_{p}$ denotes the unique radial solution of the Lane-Emden problem with $m$ nodal zones and positive at the origin. The interested reader can find more details in [21, 8, 29] and the references therein. The strategy summarized in Section 3 applies also to the Hénon problem (see [3]), indeed the Morse index of $u_{\alpha, p}$ is equal to the number of the negative eigenvalues $\widehat{\Lambda}^{\alpha}(p)$ of

$$
-\Delta \phi-V_{\alpha, p}(x) \phi=\widehat{\Lambda}^{\alpha}(p) \frac{\phi}{|x|^{2}}, \quad \phi \in \mathcal{H}_{0}(B),
$$

where now

$$
V_{\alpha, p}(x)=p|x|^{\alpha}\left|u_{\alpha, p}(x)\right|^{p-1} .
$$


Moreover, similarly as in (3.6), the negative eigenvalues $\widehat{\Lambda}^{\alpha}(p)$ of (6.3) can be decomposed as

$$
\widehat{\Lambda}^{\alpha}(p)=k^{2}+\nu^{\alpha}(p),
$$

where $\nu^{\alpha}(p)$ are the eigevalues of the following singular Sturm-Liouville problem

$$
\left\{\begin{array}{l}
-\left(r \varphi^{\prime}\right)^{\prime}=r\left(V_{\alpha, p}+\frac{\nu^{\alpha}(p)}{r^{2}}\right) \varphi \quad \text { as } 0<r<1 \\
\phi \in \mathcal{H}_{0, \mathrm{rad}}
\end{array}\right.
$$

Using the transformation $t=r^{\frac{2+\alpha}{2}}$ one sees that $\varphi_{\alpha, p}$ is an eigenfunction for (6.5) related to $\nu^{\alpha}(p)$ if and only if $\psi_{p}(t)=\varphi_{\alpha, p}(r)$ is an eigenfunction for (3.7) related to the eigenvalue

$$
\nu(p)=\left(\frac{2}{2+\alpha}\right)^{2} \nu^{\alpha}(p),
$$

see [8, Corollary 4.6]. Therefore all the results in Sections 3.2, 3.3 can be extended also to the Henon problem, in particular

$$
\nu_{1}^{\alpha}(p)<\nu_{2}^{\alpha}(p)<\ldots \nu_{m-1}^{\alpha}(p)<-\left(\frac{2+\alpha}{2}\right)^{2}<\nu_{m}^{\alpha}(p)<0,
$$

and so

$$
\mathrm{m}\left(u_{\alpha, p}\right)=m+2 \sum_{j=1}^{m}\left\lceil\sqrt{-\nu_{j}^{\alpha}(p)}-1\right\rceil=m+2 \sum_{j=1}^{m}\left\lceil\frac{2+\alpha}{2} \sqrt{-\nu_{j}(p)}-1\right\rceil,
$$

for any $p>1$.

Proof of (1.12) and (1.13). The claim follows by inserting the limits computed in Theorem 4.2 inside the Morse index formula (6.8). When $j=m$, using also (3.12), one sees that $\sqrt{-\nu_{j}(p)} \rightarrow 1$ from below, hence

$$
\left\lceil\frac{2+\alpha}{2} \sqrt{-\nu_{m}(p)}-1\right\rceil=\left\lceil\frac{\alpha}{2}\right\rceil
$$

for large values of $p$, since the ceiling function is lower semicontinuous and piecewise constant. Notice that, unlike the Lane-Emden case, also the last eigenvalue $\nu_{m}^{\alpha}(p)=\left(\frac{2+\alpha}{2}\right)^{2} \nu_{m}(p)$ gives a contribution to the Morse index. When $j=1, \ldots m-1$ it is only known that

$$
\frac{2+\alpha}{2} \sqrt{-\nu_{j}(p)} \rightarrow \frac{2+\alpha}{4} \theta_{m-j} .
$$

If the quantity on the right-hand side is non-integer, it follows that

$$
\left\lceil\frac{2+\alpha}{2} \sqrt{-\nu_{j}(p)}-1\right\rceil=\left\lceil\frac{(2+\alpha) \theta_{m-j}}{4}-1\right\rceil=\left[\frac{(2+\alpha) \theta_{m-j}}{4}\right]
$$

for large values of $p$, and formula (1.12) follows. Otherwise, only the estimate (1.13) can be deduced.

Remark 6.1 (Optimal lower bound for the Morse index). Notice that the Morse index grows quadratically with respect to $m$ : indeed in the case $\alpha=0$ (1.14) holds, and in the case $\alpha>0$ we have that

$$
\begin{aligned}
m\left(u_{\alpha, p}\right) & \geq m+2\left\lceil\frac{\alpha}{2}\right\rceil+2 \sum_{k=1}^{m-1}\left[\frac{2+\alpha}{4} \theta_{k}\right]-2(m-1) \\
& \geq m+2\left\lceil\frac{\alpha}{2}\right\rceil+2 \sum_{k=1}^{m-1}\left[\frac{\theta_{k}}{2}\right\rceil\left(1+\left\lceil\frac{\alpha}{2}\right]\right)-2(m-1) \\
& =m+\left(m\left(u_{p}\right)-m_{\text {rad }}\left(u_{p}\right)\right)\left(1+\left\lceil\frac{\alpha}{2}\right]\right)+2\left(\left\lceil\frac{\alpha}{2}\right\rceil-m+1\right),
\end{aligned}
$$


where $u_{p}$ denotes the radial solution to the Lane-Emden problem with the same number of nodal zones.

As already recalled, the lower bound (1.3) is not optimal for the Hénon problem, even in dimension $N \geq 3$. In dimension 2 that lower bound has been recently improved in [15], by exploiting the monotonicity of the Morse index with respect to the parameter $\alpha$, obtaining that

$$
m\left(u_{\alpha, p}\right) \geq m+\left(m\left(u_{0, p}\right)-m_{\mathrm{rad}}\left(u_{0, p}\right)\right)\left(1+\left[\frac{\alpha}{2}\right]\right),
$$

for any fixed $p>1$ and $\alpha>0$. The estimate (6.9) shows that neither the lower bound (6.10) is reached for large values of $p$, at least when $\alpha>2(m-1)$.

\section{Further RESUlts}

We collect here some further consequences of Theorems 4.2 and 1.1 that, in our opinion, can bring to a better understanding of both the Lane-Emden and the Hénon problem in planar domains.

7.1. Symmetric Morse index. The decomposition technique used for computing the Morse index allow also to compute suitable symmetric Morse indexes of radial solutions and so, by Morse index comparison, to distinguish among radial solutions and least energy solutions in suitable symmetric spaces, in the spirit of [23. The key point is that not only the eigenvalues but also the associated eigenfunctions of the singular eigenvalue problem (3.5) decompose, indeed in radial coordinates they can be written as

$$
\psi_{j, p}(r)(A \cos (k \theta)+B \sin (k \theta))
$$

where

- $\psi_{j, p}$ is a solution to the singular Sturm-Liouville problem (3.7) related to $\nu_{j}(p)$,

- $\cos (k \theta), \sin (k \theta)$ are the eigenfunctions of the Laplace-Beltrami operator on the circle, related to the eigenvalue $k^{2}$.

Explicit formulas computing the Morse index in symmetric spaces by means of the singular eigenvalues can be found in [3, Corollaries 4.3, 4.11]. The symmetric Morse index can be computed then, for large values of the parameter $p$, by exploiting Theorem 4.2 .

7.2. Nondegeneracy for large values of $p$. It is well known that the radial solutions are radially non-degenerate, meaning that the linearized problem

$$
-\Delta w=p|x|^{\alpha}\left|u_{\alpha, p}\right|^{p-1} w
$$

does not have nontrivial solutions in $H_{0, \operatorname{rad}}(B)$ (see [28 for $\alpha=0$ and 8 for $\alpha>0$ ). Nonradial degeneracy (i.e. existence of solutions in $H_{0}(B) \backslash H_{0, \text { rad }}(B)$ ), on the other hand, can be characterized in terms of the singular eigenvalues through the condition

$$
\nu^{\alpha}(p)=-k^{2}
$$

see [3, Proposition 1.3]. So Theorem 4.2 together with (2.4), yields also that

Corollary 7.1. For every positive integer $m$, there exists $p^{*}>1$ such that radial solutions to the Lane-Emden problem (2.1) with $m$ nodal zones are nondegenerate for $p>p^{*}$.

Moreover for every positive integer $m$ and for every $\alpha>0$ except at most the sequences $\frac{4 n}{\theta_{i}}-2$ (for $i=1, \ldots m-1, n \in \mathbb{N}$ ), there exists $p^{*}>1$ such that radial solutions to the Hénon problem (6.1) with $m$ nodal zones are nondegenerate for $p>p^{*}$. 
7.3. Bifurcation. Observe that Theorem 1.1 gives the values of the Morse index for $p$ large. On the other side one can also compute the Morse index when $p$ is close to 1 , by exploiting the (much easier to derive) asymptotic behavior of the radial solutions as $p \rightarrow 1$ from the right (see for instance [13, 24]), and characterizing it in terms of zeros of suitable Bessel functions of the first kind (see [23] for the case $(\alpha, N, m)=(0,2,2)$ and 4 for the general case).

As a consequence one can now detect values of $p \in(1+\infty)$ where the Morse index changes. This is of course a sufficient condition for degeneracy of the solutions at those values of $p$, which could convey to bifurcation from the curve $p \mapsto u_{\alpha, p}$, for each radial solution $u_{\alpha, p}$. We refer to 23 for the case $(\alpha, N, m)=(0,2,2)$ where 3 branches of bifurcation have been detected, due to a change in the Morse index caused by the first eigenvalue $\nu_{1}(p)$. For solutions with more nodal regions other eigenvalues may play a role. To give an idea of what may happen, let us consider for instance the case of the solution $u_{p}$ of the planar Lane-Emden problem $(\alpha=0)$ with $m=3$ nodal regions. From [4] we know that in this case, for $p$ close to 1

$$
\nu_{1}(p) \in\left(-5^{2},-4^{2}\right), \quad \nu_{2}(p) \in\left(-3^{2},-2^{2}\right), \quad \nu_{3}(p) \in(-1,0)
$$

while, from Theorem 1.2 one deduces that for $p$ large

$$
\nu_{1}(p) \in\left(-10^{2},-9^{2}\right), \quad \nu_{2}(p) \in\left(-6^{2},-5^{2}\right), \quad \nu_{3}(p) \in(-1,0) .
$$

As a consequence it follows that

$$
m\left(u_{p}\right)=\left\{\begin{array}{lr}
15 & \text { for } p \text { close to } 1 \\
31 & \text { for } p \text { large }
\end{array}\right.
$$

respectively, and moreover there exist $p=p_{k}>1$ for $k=3,4,5$ and $p=\hat{p}_{k}>1$ for $k=5,6,7,8,9$ at which the degeneracy condition (7.2) is satisfied as follows:

$$
\begin{array}{ll}
\nu_{2}\left(p_{k}\right)=-k^{2}, & \text { for } k=3,4,5 \\
\nu_{1}\left(\hat{p}_{k}\right)=-k^{2}, & \text { for } k=5,6,7,8,9,
\end{array}
$$

thus involving the first two eigenvalues $\nu_{1}(p)$ and $\nu_{2}(p)$. Those $p_{k}, \hat{p}_{k}$ are the values of $p$ at which one expects that $u_{p}$ bifurcates. In [19] some numerical results in this direction have been indeed obtained, see also [2, Proposition 4.5] where bifurcations at $\hat{p}_{k}$ (hence from the first eigenvalue) is proved.

\section{REFERENCES}

[1] A. Aftalion and F. Pacella. Qualitative properties of nodal solutions of semilinear elliptic equations in radially symmetric domains. Compt. Rendus Math., 339:339, 2004.

[2] A. L. Amadori. Global bifurcation for the Hénon problem. Communications on Pure and Applied Analysis, 19(10), 2020.

[3] A. L. Amadori and F. Gladiali. On a singular eigenvalue problem and its applications in computing the Morse index of solutions to semilinear PDE's. Nonlinear Analysis: Real World Applications, 55:103133, 2020.

[4] A.L. Amadori. On the asymptotically linear Hénon problem. Communications in Contemporary Mathematics, Article number 2050042, 2020.

[5] A.L. Amadori and F. Gladiali. Bifurcation and symmetry breaking for the Hénon equation. Advances in Differential Equations, 19(7/8):755-782, 2014.

[6] A.L. Amadori and F. Gladiali. Asymptotic profile and morse index of nodal radial solutions to the Hénon problem. Calculus of Variations and Partial Differential Equations, 58(5):1-47, September 2019.

[7] A.L. Amadori and F. Gladiali. The Hénon problem with large exponent in the disc. Journal of Differential Equations, 268(10):5892-5944, 2020.

[8] A.L. Amadori and F. Gladiali. On a singular eigenvalue problem and its applications in computing the Morse index of solutions to semilinear PDE's: part II. Nonlinearity, 33(6):2541-2561, apr 2020.

[9] F.V. Atkinson and L.A. Peletier. Elliptic equations with nearly critical growth. Journal of Differential Equations, 70(3):349-365, 1987.

[10] T. Bartsch and M. Degiovanni. Nodal solutions of nonlinear elliptic Dirichlet problems on radial domains. Atti Accad. Naz. Lincei Cl. Sci. Fis. Mat. Natur. Rend. Lincei (9) Mat. Appl., 17:69, 2006. 
[11] T. Bartsch and T. Weth. A note on additional properties of sign changing solutions to superlinear equations. Topol. Methods Nonlinear Anal., 22:1, 2003.

[12] T. Bartsch and M. Willem. Infinitely many radial solutions of a semilinear elliptic problem on $\mathbb{R}^{N}$. Archive for Rational Mechanics and Analysis, 124(3):261-276, 1993.

[13] D. Bonheure, V. Bouchez, C. Grumiau, and J. Van Schaftingen. Asymptotics and symmetries of least energy nodal solutions of lane-emden problems with slow growth. Communications in Contemporary Mathematics, 10(4):609-631, 2008.

[14] C.C. Chen and C.S-Lin. Mean field equations of liouville type with singular data: sharper estimates. Discrete Cont. Dyn. Syst., 28(3):1237-1272, 2010.

[15] W. L. da Silva and E. M. dos Santos. Monotonicity of the Morse index of radial solutions of the Hénon equation in dimension two. Nonlinear Analysis: Real World Applications, 48:485-492, 2019.

[16] F. De Marchis, I. Ianni, and F. Pacella. Exact Morse index computation for nodal radial solutions of Lane-Emden problems. Mathematische Annalen, 367(1):185-227, 2017.

[17] F. De Marchis, I. Ianni, and F. Pacella. A Morse index formula for radial solutions of Lane-Emden problems. Advances in Mathematics, 322:682-737, 2017.

[18] E. M. dos Santos and F. Pacella. Morse index of radial nodal solutions of Hénon type equations in dimension two. Commun. Contemp. Math., 19, 2017.

[19] B. Fazekas, F. Pacella, and M. Plum. Approximate nonradial solutions for the lane-emden problem in the ball. Preprint.

[20] P. Figueroa and S. L. N. Neves. Nonradial solutions for the Hénon equation close to the threshold. Advanced Nonlinear Studies, 2019

[21] F. Gladiali, M. Grossi, and S.L.N. Neves. Symmetry breaking and morse index of solutions of nonlinear elliptic problems in the plane. Communications in Contemporary Mathematics, 18(5), 2016.

[22] F. Gladiali, M. Grossi, F. Pacella, and P.N. Srikanth. Bifurcation and symmetry breaking for a class of semilinear elliptic equations in an annulus. Calculus of Variations and Partial Differential Equations, 40(3):295-317, 2011.

[23] F. Gladiali and I. Ianni. Quasi-radial solutions for the Lane-Emden problem in the ball. Nonlinear Differential Equations and Applications NoDEA, 27(2):13, 2020.

[24] M. Grossi. On the shape of solutions of an asymptotically linear problem. Annali della Scuola Normale - Classe di Scienze, 8(3):429-449, 2009.

[25] M. Grossi, C. Grumiau, and F. Pacella. Lane Emden problems with large exponents and singular Liouville equations. Journal des Mathematiques Pures et Appliquees, 101(6):735-754, 2014.

[26] M. Grossi, A. Saldaña, and H. Tavares. Sharp concentration estimates near criticality for radial signchanging solutions of dirichlet and neumann problems. Proceedings of the London Mathematical Society, 120(1):39-64, 2020.

[27] M. Hénon. Numerical experiments on the stability of spherical stellar systems. Astronom. Astrophys., $24: 229,1973$

[28] A. Harrabi, S. Rebhi, and A. Selmi. Existence of radial solutions with prescribed number of zeros for elliptic equations and their Morse index. Journal of Differential Equations, 251(9):2409 - $2430,2011$.

[29] I. Ianni and A. Saldaña. Sharp asymptotic behavior of radial solutions of some planar semilinear elliptic problems. arXiv preprint arXiv:1908.10503, 2019.

[30] R. Kajikiya. Sobolev norm of radially symmetric oscillatory solutions for super-linear elliptic equations. Hiroshima Math. J., 20:259-276, 1990.

[31] J. Kubler and T. Weth. Spectral asymptotics of radial solutions and nonradial bifurcation for the Hénon equation. Discrete Contin. Dyn. Syst., Ser., 40:3629, 2019.

[32] S.S. Lin. Asymptotic behavior of positive solutions to semilinear elliptic equations on expanding annuli. Journal of Differential Equations, 120(2):255-288, 1995.

[33] W. M. Ni and R. D. Nussbaum. Uniqueness and nonuniqueness for positive radial solutions of $\Delta u+$ $f(u, r)=0$. Comm. Pure Appl. Math., 38:67, 1985.

[34] W. N. Ni. A nonlinear Dirichlet problem on the unit ball and its applications. Indiana Univ. Math. J., $31: 801,1982$

[35] Willem M. Smets D. and J. Su. Non-radial ground states for the Hénon equation. Commun. Contemp. Math., 4:467, 2002. 
(Anna Lisa Amadori) Dipartimento di Scienze Applicate, Università di Napoli "Parthenope", Centro Direzionale di Napoli, Isola C4, 80143 Napoli, Italy.

Email address: annalisa.amadori@uniparthenope.it

(Francesca De Marchis) Dipartimento di Matematica Guido Castelnuovo, Sapienza Università di Roma, Piazzale Aldo Moro 5, 00185 Roma

Email address: demarchis@mat.uniroma1.it

(Isabella Ianni) Dipartimento SBAI, Sapienza Università di Roma, via Scarpa 10, 00161 Roma

Email address: isabella.ianni@uniroma1.it 